\title{
A New Approach to Dimensioning Optical Networks
}

\author{
Tapan Kumar Nayak, Student Member, IEEE, and Kumar N. Sivarajan, Member, IEEE
}

\begin{abstract}
A network dimensioning method allocates appropriate capacities to the links based on the network topology and traffic requirements. In this paper, we introduce the concept of absorption probability instead of blocking probability which is a more appropriate measure to express the grade of service of an optical network, in many cases. Absorption probability can be obtained from transient analysis of a Markov chain. Computation of exact absorption probabilities requires large computing resources and is thus feasible only for small networks. Here, we present a method to approximate the absorption probability of a wavelength-routed network with arbitrary topology and traffic patterns assuming the nodes have full wavelength conversion capability. We show that the approximation method performs well especially in the desired range of absorption probabilities and it is computationally efficient.
\end{abstract}

Index Terms-Absorption probability, capacity allocation, network dimensioning, optical networks.

\section{INTRODUCTION}

W AVELENGTH-DIVISION multiplexing (WDM) technology for optical networks adds a new dimension to the field of telecommunication and it offers the capability of building very large wide-area networks with throughputs on the order of gigabits per second for each node [1]. In this work we consider that all the nodes have full wavelength conversion capability and a call requesting a multihop route can be assigned different wavelengths on different links of the requested path. Wavelength conversion in a WDM network offers a reduction in blocking arising from wavelength contentions. Optical wavelength converters are still in an experimental stage; however, with electronic wavelength crossconnects (WXC), it is easier to realize wavelength conversion [2]. With full wavelength conversion capability, an optical network is equivalent to a conventional circuit switched network.

The key aspect of designing a wavelength routing network is determining the number and, more generally, the set of wavelengths which must be provided on each DWDM link. We call this the wavelength dimensioning problem. Many network dimensioning methods are based on mathematical models with cost minimization as the objective and grade-of-service constraints expressed in terms of end-to-end blocking probabilities. The models turn out to be nonlinear optimization problems with a rather large number of variables and in some formulations many nonlinear constraints. A straightforward solution method

Manuscript received February 18, 2001; revised July 30, 2001. The work of T. K. Nayak was supported by a fellowship from IBM India Research Lab, New Delhi. The work of K. N. Sivarajan was supported by the Department of Science and Technology, Government of India, through the Swarnajayanti fellowship.

T. K. Nayak is with the Indian Institute of Science, Bangalore, India (e-mail: tapan@protocol.ece.iisc.ernet.in).

K. N. Sivarajan is with Tejas Networks, Bangalore, India (e-mail: kumar@ tejasnetworks.com).

Publisher Item Identifier S 0733-8716(02)00161-0. is to use the algorithms for nonlinear constrained minimization [3]-[5]. Several network dimensioning methods are proposed and studied through simulations in the literature [6], [7]. Computational complexities and relative merits for different models are discussed in [8] and [9]. Heuristic methods for large scale networks are also proposed [10]. For a network dimensioning model, most of the computation time is spent in calculating a direction to search for an optimal solution, which in turn requires the evaluation of gradients of the constraint and objective functions at each iteration. The method used to compute the gradients of the constraint functions can significantly influence the overall computation time for a network-dimensioning problem and it involves the computation of end-to-end blocking probabilities of the links for different values of capacity.

For a large circuit-switched network, Kelly proposed an efficient method to estimate the loss probabilities, based on solving a fixed point problem involving Erlang's formula under the assumption of independent blocking, and showed that under a limiting regime the estimates of loss probabilities converge to the correct values [11]. There are efficient methods to approximate blocking probabilities for all-optical networks with no wavelength conversion [12], [13] and also with limited wavelength conversion [14]. These network dimensioning methods are based on blocking probabilities which are computed assuming the network is in a steady state condition.

\section{Problems With the Blocking Model}

In optical networks lightpaths carry data at very high bit rates and are typically set up on a provisioning basis. Lightpaths in optical networks are long-lived and the cost of deploying a lightpath is very high compared to the cost of deploying a circuit in other networks, for example, telephone networks. Blocking probabilities are calculated based on the steady state model of a network. Networks with long-lived lightpaths may not reach a steady state because the demands for lightpaths may change after some time. Even if the network reaches a steady state, it may take a long time to do so resulting in initial overdimensioning of the network and higher upfront expenditure. So for dimensioning of optical networks, the blocking model is inefficient because it allocates excessively large capacities right from the beginning. Apart from this, the blocking probability is calculated based on the a priori estimate of traffic on each route. But the traffic can vary after a certain period of time, for example, six months or one year, which requires recalculation of blocking probabilities and reallocation of capacities.

So the blocking model may not be appropriate for dimensioning optical networks where, rather than block a lightpath request, an operator is likely to add more capacity in his network to support the request [2]. In this paper we consider a traffic growth 


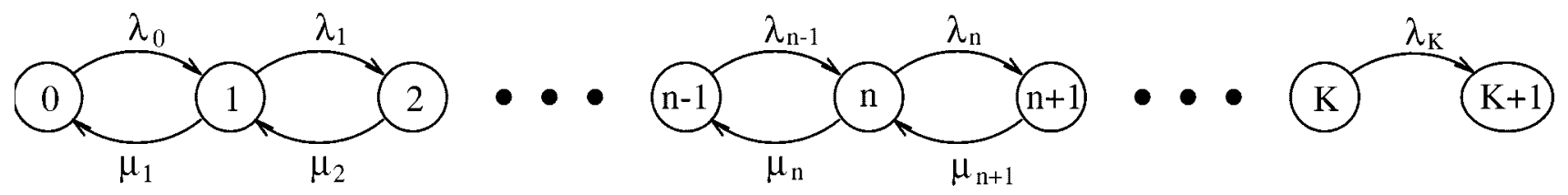

Fig. 1. Markov chain of the busy wavelength distribution on a single link.

model and introduce the concept of absorption probability instead of blocking probability to express the grade of service of a network. The absorption probability at time $t$ is the probability that at least one lightpath request will be blocked before time $t$. So rather than designing a network to achieve a certain blocking probability, it may be more appropriate to design it to achieve a certain absorption probability over some time period, say six months or one year. This is a time period for which the network has been dimensioned and the network capacity will need to be upgraded before the end of this period. This provides a model to dimension the network.

We model the network by a Markov chain. In the terminology of Markov chains, the state from which no exit is possible is called an absorbing state, and the probability of staying at an absorbing state is called the absorption probability. We elaborate on this traffic model in the next section.

\section{TRAFFIC MODEL AND OVERVIEW}

In our model, the network is assumed to start with no lightpaths at all. This is quite appropriate in the case of a newly built optical network. Lightpaths are assumed to arrive randomly according to a statistical model and have to be set up on the optical layer. Some lightpaths may depart as well but eventually we will have to reject a lightpath request. We are interested in dimensioning the WDM links so that the first lightpath request rejection will occur, with high probability, after a specified period of time $T$. This is a reasonable model today since lightpaths are long-lived. This longevity, combined with the cost of a high bandwidth lightpath today, means that a network operator is unlikely to reject a lightpath request. Rather, he would like to upgrade his network by the addition of more capacity on existing links in order to accommodate the lightpath request. The time period $T$ corresponds approximately to the time by which the operator must institute such upgrades in order to avoid rejecting lightpath requests. So if a network has an absorption probability of $p$ at time $T$, it means the operator has to add additional capacity with probability $p$ by time $T$ in order to be able to set up all the requested lightpaths/connections.

For an illustration of our results, consider the network shown in Fig. 13. This is a 20-node, 32-link network representing a skeleton of the ARPANET. An average of one lightpath request is assumed to arrive every month, between every pair of nodes, and this lightpath is assumed to be in place for an average of one year. The link capacities shown are calculated such that no link will need a capacity upgrade within six months, with high (90\%) probability. In this example, the network has an absorption probability $p<0.1$ at time $T=6$ months.

For this traffic model, as with many others including the more commonly employed blocking model, the analysis problem is easier to solve than the design problem. It is easier to calculate the first passage time at which the network operator will have to block a lightpath request for given link capacities than it is to design the link capacities to achieve a prespecified first-passage time. However, the capacity design or dimensioning problem can be solved by iterating on the analysis problem. We can calculate the absorption probabilities for a given set of capacities, and if these are not acceptable on some links, increase the capacities of those links and recalculate the absorption probabilities. We illustrate this aspect at the end of the paper with reference to the network and capacities shown in Fig. 13. For the rest of the paper, we will focus on the estimation of absorption probabilities.

Estimation of absorption probability is based on the transient analysis of the Markov chain model of the network. In this paper we introduce a very efficient method to compute the absorption probabilities for any network which shows excellent performance even for large networks. We show that the estimates of absorption probabilities converge in a limiting regime.

The rest of the paper is organized as follows. In Section IV, we consider a single link network and compute its absorption probability. In Section V, we consider a two-link network and present the methods for approximation of absorption probability together with the lower and upper bounds. Computation of the exact absorption probability for this case is discussed in the Appendix. In Section VI, we consider a large network, outline the traffic assumptions, and describe our method for calculation of approximate absorption probabilities in large networks. We present our results in Section VII and conclude in Section VIII.

\section{SINGLE LINK}

In our proposed method, the approximate absorption probability of a route in a large network is calculated based upon the individual absorption probabilities of the corresponding links with the assumption that the links are independent. The details of the method are discussed in Section VI. This motivates us to study a single link network extensively and this will help to calculate the absorption probability for a larger network.

In this section we consider a single link network of capacity $K$. The busy wavelength distribution in the link can be modeled by a birth-death process where a state of the Markov chain is defined by the number of busy wavelengths in the link.

Let $P_{n}(t)$ be the probability of state $n$ at time $t$. Arrivals are assumed to be Poisson with state-dependent arrival rate $\lambda_{n}$ for transition from state $n$ to state $n+1$. Departures are also assumed Poisson with state-dependent departure rate $\mu_{n}$ for transition from state $n$ to state $n-1$. The birth and death model for a single link with the above assumptions is shown in Fig. 1. 
We then have the following differential equations [15]. We assume the initial state is zero

$$
\begin{aligned}
P_{n}^{\prime}(t)= & -\left(\lambda_{n}+\mu_{n}\right) P_{n}(t)+\lambda_{n-1} P_{n-1}(t) \\
& +\mu_{n+1} P_{n+1}(t) \text { for } 1 \leq n \leq K-1 \\
P_{0}^{\prime}(t)= & -\lambda_{0} P_{0}(t)+\mu_{1} P_{1}(t) \\
P_{K}^{\prime}(t)= & -\left(\lambda_{K}+\mu_{K}\right) P_{K}(t)+\lambda_{K-1} P_{K-1}(t) \\
P_{K+1}^{\prime}(t)= & \lambda_{K} P_{K}(t) .
\end{aligned}
$$

Initial Condition: $P_{0}(0)=1, P_{n}(0)=0$ for $n \geq 1$. Define

$$
\mathbf{A}=\left[\begin{array}{cccccc}
-\lambda_{0} & \mu_{1} & \ldots & 0 & 0 & 0 \\
\lambda_{0} & -\left(\lambda_{1}+\mu_{1}\right) & \ldots & 0 & 0 & 0 \\
\vdots & \vdots & & \vdots & \vdots & \vdots \\
0 & 0 & \ldots & \lambda_{K-1} & -\left(\lambda_{K}+\mu_{K}\right) & 0 \\
0 & 0 & \ldots & 0 & \lambda_{K} & 0
\end{array}\right]
$$$$
\mathbf{P}(t)=\left[\begin{array}{c}
P_{0}(t) \\
P_{1}(t) \\
\vdots \\
P_{K}(t) \\
P_{K+1}(t)
\end{array}\right] \quad \text { and } \quad \mathbf{P}(0)=\left[\begin{array}{c}
1 \\
0 \\
\vdots \\
0 \\
0
\end{array}\right]
$$

Now, the system can be written in the following form:

$$
\mathbf{P}^{\prime}(t)=\mathbf{A P}(t)
$$

where $\mathbf{A}$ is a $(K+2) \times(K+2)$ constant matrix.

The link will be absorbed if $K$ wavelengths are busy and another arrival occurs. In the terminology of Markov chains the $(K+1)$-th state is an absorbing state from which no exit is possible. From (4) it follows that $P_{K+1}^{\prime}(t) \geq 0$ as $\lambda_{K}>0$ and $0 \leq P_{K}(t) \leq 1$. So, $P_{K+1}(t)$ is a monotonically nondecreasing function of $t$ with $P_{K+1}(0)=0$.

The solution of the absorption probability $P_{K+1}(t)$ for a single link network can be obtained explicitly as a function of $t$ and the method of solution is discussed in Appendix A. The explicit form of the solution is

$$
P_{K+1}(t)=1-\sum_{r=1}^{K+1} a_{r} \cdot e^{\alpha_{r} t}
$$

where $\alpha_{1}, \alpha_{2}, \ldots \alpha_{K+1}$ are the $(K+1)$ negative eigenvalues of matrix $\mathbf{A}$ arranged in decreasing order and $a_{1}, a_{2}, \ldots a_{K+1}$ are the corresponding coefficients.

From (6), it is observed that the probability of ultimate $a b$ sorption $P_{K+1}(\infty)=1$.

If the dominant eigenvalue $\alpha_{1}$ is well separated from the other eigenvalues, for sufficiently large values of $t$

$$
P_{K+1}(t) \approx 1-a_{1} e^{\alpha_{1} t} .
$$

The approximate absorption probabilities considering a single dominant eigenvalue and two dominant eigenvalues are plotted with the exact absorption probability with state independent arrival rate $\lambda_{n}=\lambda$ and $\mu_{n}=n \mu$, for all $n$ in Fig. 2. Note that

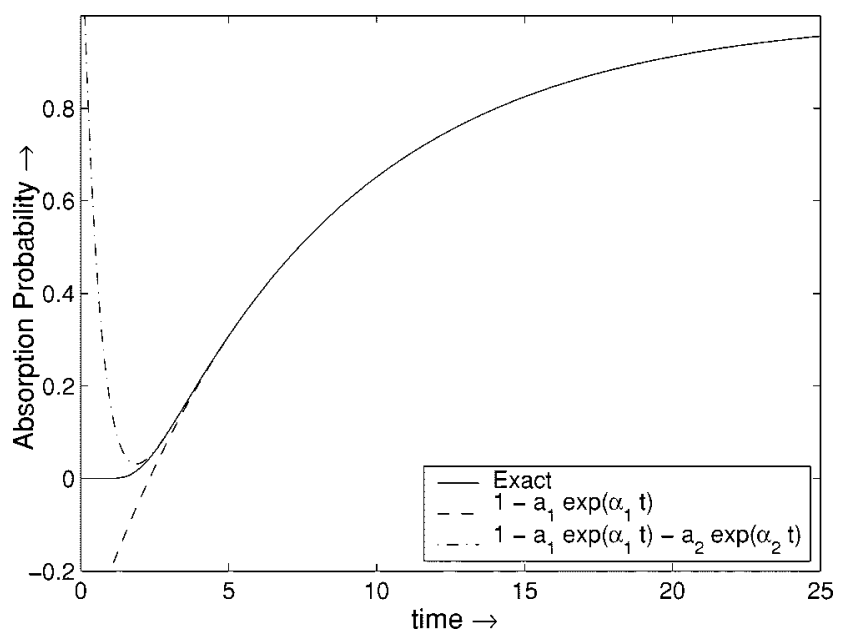

Fig. 2. Exact and approximate absorption probabilities of a single link network with capacity $K=32$, arrival rate $\lambda=24$, and mean service time $1 / \mu=1$.

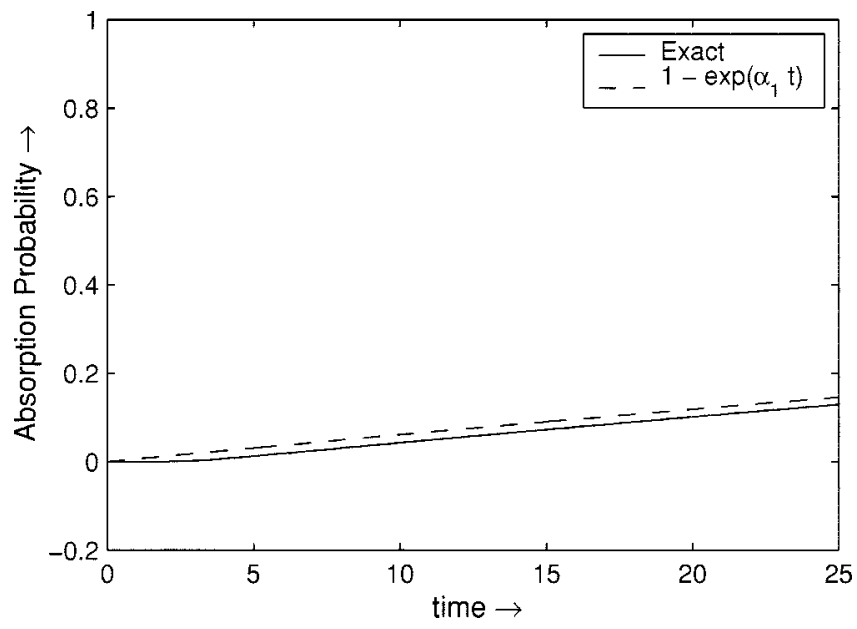

Fig. 3. Exact and approximate absorption probability with capacity $K=32$, arrival rate $\lambda=18$, and mean service time $1 / \mu=1$.

the approximations are very good for sufficiently large values of time $t$.

Define $\rho=\lambda / \mu$ with $\lambda_{n}=\lambda$ and $\mu_{n}=n \mu$, for all $n$. If $\rho$ is sufficiently less than $K+1$, the dominant eigenvalue $\alpha_{1}$ is very close to zero and in this case (see Appendix A)

$$
P_{K+1}(t) \approx 1-e^{\alpha_{1} t} .
$$

The exact absorption probability and the approximate absorption probability $1-e^{\alpha_{1} t}$ are plotted in Fig. 3. Observe that the approximation is very good for $\rho=18$ when the capacity $K$ is 32.

So in certain conditions, the first passage time can be well approximated by an exponential random variable with mean $-1 / \alpha_{1}$.

For the rest of the discussion, we will usually assume that $\mu=1$. Therefore, time $t$ is measured in units of a connection holding time. This time is expected to be large, of the order of several months or a few years in optical networks. Therefore, the values of $t$ that will be of interest are usually small (0.5-2). 


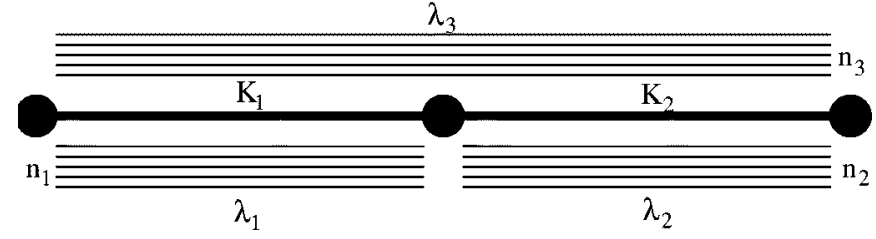

Fig. 4. Different types of calls in a two-link network.

\section{TWO-LINK NETWORK}

In this section we consider a very simple network, a two-link optical network. Here we will compute both the exact and approximate absorption probabilities and compare the results.

For a two-link network, let $\tau_{1}$ and $\tau_{2}$ be the first passage times for link-1 and link-2, respectively. If we assume both $\tau_{1}$ and $\tau_{2}$ are exponentially distributed with means $1 / \beta_{1}$ and $1 / \beta_{2}$ respectively, then

$$
\tau_{i} \sim \exp \left(\beta_{i}\right) \Rightarrow P\left(\tau_{i} \leq t\right)=1-e^{-\beta_{i} t}\left(\beta_{i}>0, i=1,2\right) .
$$

Let $\tau$ be the first passage time of the whole network. Then

$$
\tau=\min \left(\tau_{1}, \tau_{2}\right) \quad \text { and } \quad P(\tau>t)=P\left(\tau_{1}>t, \tau_{2}>t\right) .
$$

Let us consider the following two cases [16].

1) The first passage times $\tau_{1}$ and $\tau_{2}$ are independent

$$
\begin{gathered}
P(\tau>t)=P\left(\tau_{1}>t\right) \cdot P\left(\tau_{2}>t\right) \\
=e^{-\beta_{1} t} \cdot e^{-\beta_{2} t}=e^{-\left(\beta_{1}+\beta_{2}\right) t} \\
\Rightarrow P(\tau \leq t)=1-e^{-\left(\beta_{1}+\beta_{2}\right) t} \\
\therefore \tau \sim \exp \left(\beta_{1}+\beta_{2}\right) .
\end{gathered}
$$

2) $\tau_{1}$ and $\tau_{2}$ are linearly dependent. So there exists some constant $a$, so that $\tau_{2}=a \tau_{1}$

$$
\begin{gathered}
E\left(\tau_{2}\right)=a E\left(\tau_{1}\right) \Rightarrow 1 / \beta_{2}=a / \beta_{1} \Rightarrow \beta_{1}=a \beta_{2} \\
\tau= \begin{cases}\tau_{1} \sim \exp \left(\beta_{1}\right) & \text { if } a \geq 1 \\
\tau_{2} \sim \exp \left(\beta_{2}\right) & \text { if } a<1\end{cases} \\
\therefore \tau \sim \exp \left(\max \left(\beta_{1}, \beta_{2}\right)\right) .
\end{gathered}
$$

In the above, 1) and 2) are two extreme assumptions because either there is no correlation between the two links or they are fully correlated. Also, 2) implies that if there is an arrival in link-1, simultaneously there are also $a$ arrivals in link-2. So in the general case, $\tau_{1}$ and $\tau_{2}$ may be dependent and if $\tau$ is approximated by an exponential distribution $(\exp \beta)$, then

$$
\max \left(\beta_{1}, \beta_{2}\right) \leq \beta \leq \beta_{1}+\beta_{2} .
$$

This result will help us to obtain the upper and lower bounds on absorption probability.

For a two-link network there are three typea of calls which are all shown in Fig. 4. Here, we state the assumptions about the network and traffic that are used for computation of absorption probability of a two-link optical network with full wavelength conversion capability.

\section{A. Assumptions}

1) Capacities of link-1 and link-2 are $K_{1}$ and $K_{2}$, respectively.
2) Arrivals are Poisson with state-independent arrival rate $\lambda_{i}$ for type-i calls, $i=1,2,3$.

3) Duration of each call is exponentially distributed with unit mean.

The exact absorption probability can be calculated based upon a three-dimensional Markov chain model. The method is discussed in Appendix B.

\section{B. Bounds on Absorption Probability}

Let us define a time-dependent function $f$ so that $f(\lambda, K, t)$ is the no absorption probability of a single link of capacity $K$ with state independent arrival rate $\lambda$ and mean service time unity at time $t$ and it can be obtained from (6) as $1-P_{K+1}(t)$.

1) Upper Bound: Previously, we discussed that if we consider the links independently it will yield an upper bound on absorption probability.

Let us define $\lambda^{(1)}=\lambda_{1}+\lambda_{3}$. Consider link-1 as an independent single link of capacity $K_{1}$ with arrival rate $\lambda^{(1)}$ and mean service time unity. So the no absorption probability of link-1 is

$$
P_{\text {noabs }}^{(1)}(t)=f\left(\lambda^{(1)}, K_{1}, t\right) \text {. }
$$

Similarly, for link-2 define $\lambda^{(2)}=\lambda_{2}+\lambda_{3}$ and the no absorption probability of link-2

$$
P_{\text {noabs }}^{(2)}(t)=f\left(\lambda^{(2)}, K_{2}, t\right) .
$$

The absorption probability of the two-link network is

$$
P_{\text {abs }}(t) \leq 1-\left(P_{\text {noabs }}^{(1)}(t) \cdot P_{\text {noabs }}^{(2)}(t)\right) \text {. }
$$

2) Lower Bound: Let us define

$$
\begin{aligned}
K_{l} & =\max \left(K_{1}, K_{2}\right) \\
\lambda^{(l)} & =\max \left(\lambda_{1}+\lambda_{3}, \lambda_{2}+\lambda_{3}\right) .
\end{aligned}
$$

Consider a single link with capacity $K_{l}$ with state-independent arrival rate $\lambda^{(l)}$ and mean service time unity. The no absorption probability is

$$
P_{\text {noabs }}^{(l)}(t)=f\left(\lambda^{(l)}, K_{l}, t\right) .
$$

The absorption probability of the two-link network is

$$
P_{\text {abs }}(t) \geq 1-P_{\text {noabs }}^{(l)}(t) .
$$

Again, type- 1 and type- 2 calls are independent in all cases. So this can yield another lower bound as follows. Let

$$
\begin{aligned}
P_{\text {noabs }}^{(1 l)}(t) & =f\left(\lambda_{1}, K_{1}, t\right) \text { and } P_{\text {noabs }}^{(2 l)}(t) \\
& =f\left(\lambda_{2}, K_{2}, t\right) \\
P_{\text {abs }}(t) & \geq 1-\left(P_{\text {noabs }}^{(1 l)}(t) \cdot P_{\text {noabs }}^{(2 l)}(t)\right) .
\end{aligned}
$$

\section{Approximation of Absorption Probability}

In this section, we will describe a fixed point approximation method that will help to calculate the approximate absorption probability for a two-link network. The assumption that the links are independent will yield an upper bound of the absorption probability when $\lambda_{1}+\lambda_{3}$ and $\lambda_{2}+\lambda_{3}$ are the arrival rates for link-1 and link-2, respectively (see Section V.B.1). But the exact 
absorption probability may be far from the upper bound. To calculate the approximate absorption probability it is assumed that the links are independent but the arrival rates are thinned properly based upon the absorption probability of the other link. The carried traffic on link-1 at time $t$ is approximated by a Poisson process with arrival rate $\lambda_{1}+\lambda_{3}\left(1-P_{\mathrm{abs}}^{2}(t)\right)$ and the carried traffic on link-2 is approximated by $\lambda_{2}+\lambda_{3}\left(1-P_{\text {abs }}^{1}(t)\right)$ when $P_{\mathrm{abs}}^{i}(t)$ is the absorption probability of link- $i$ at time $t, i=1,2$.

The absorption probability of a link $j, j=1,2$ can be calculated from the following set of fixed-point equations:

$$
\begin{aligned}
& P_{\mathrm{abs}}^{1}(t)=F\left(\lambda_{1}+\lambda_{3}\left(1-P_{\mathrm{abs}}^{2}(t)\right), K_{1}\right) \\
& P_{\mathrm{abs}}^{2}(t)=F\left(\lambda_{2}+\lambda_{3}\left(1-P_{\mathrm{abs}}^{1}(t)\right), K_{2}\right)
\end{aligned}
$$

where $F(\lambda(t), K)$ is the absorption probability of a single link of capacity $K$ with time-dependent Poisson arrival rate $\lambda(t)$ and mean service time unity at time $t$. Note that the arrival rate is a function of time here. $F(\lambda(t), K)$ and the coupled set of nonlinear equations (12) and (13) can be solved by numerical techniques.

Finally, the absorption probability of the two-link network can be obtained based upon the assumption that the links are independent

$$
P_{\mathrm{abs}}(t)=1-\left(1-P_{\mathrm{abs}}^{1}(t)\right)\left(1-P_{\mathrm{abs}}^{2}(t)\right) .
$$

We present another approximation method for two-link networks based on the assumption that conditional on two-link traffic $n_{3}$, single-link traffics $n_{1}$ and $n_{2}$ are independent. The method is discussed in Appendix C.

\section{Experimental Results}

Here we present the experimental results of various techniques to compute the absorption probability as a function of time.

For a two-link network, the exact absorption probability, the upper bound, the lower bound, and the approximate absorption probabilities using two different methods, are plotted as a function of time in Fig. 5.

It is observed from Fig. 5 that the approximate absorption probabilities are very close to the exact, especially for low absorption probabilities. Observe that the fixed point approximation method gives a very good approximation up to probability 0.6 and it deviates for larger values. This behavior can be explained as follows.

For large values of absorption probability, the thinning for type- 3 calls is excessive and the thinned terms $\lambda_{3}\left(1-P_{\text {abs }}^{2}(t)\right)$ and $\lambda_{3}\left(1-P_{\text {abs }}^{1}(t)\right)$ are almost zero. This is equivalent to a two-link network with only type- 1 and type- 2 calls with arrival rates $\lambda_{1}$ and $\lambda_{2}$, respectively, and the corresponding absorption probability results in a large deviation from the exact absorption probability. But this causes no problem in designing a network, in practice, because the required absorption probability is typically low (0.1 or less).

\section{LARGE NETWORK}

In this section we are considering a general optical network with the following assumptions.

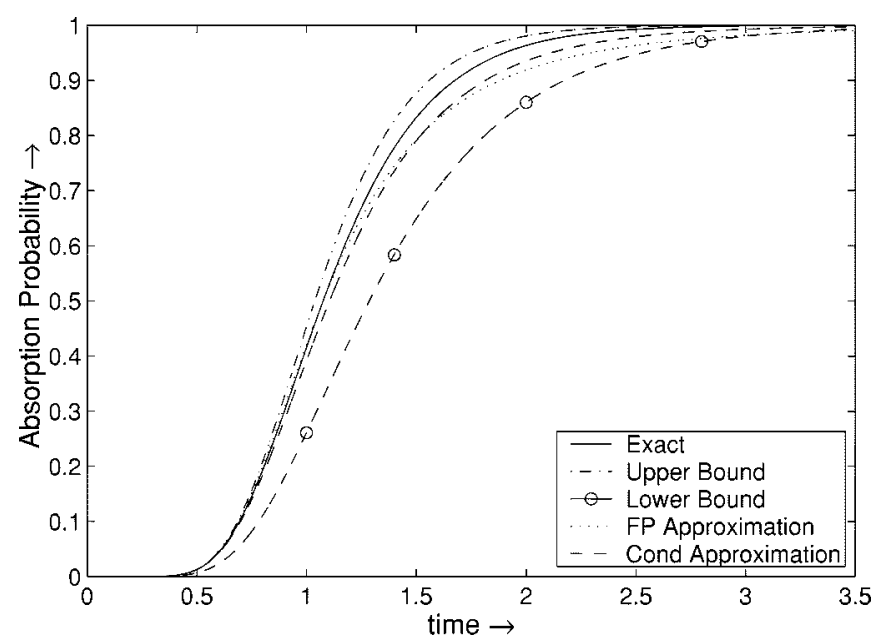

Fig. 5. Absorption probability of a two-link network with capacity $K_{1}=$ $K_{2}=16$, arrival rates $\lambda_{1}=\lambda_{2}=14, \lambda_{3}=7$, and mean service time $1 / \mu=$ 1 . Note that the approximations are very good for low absorption probabilities.

\section{A. Assumptions Regarding the Network and Offered Traffic}

1) The network consists of $J$ links connected in an arbitrary fashion. The links are labeled by $j=1,2, \ldots, J$ and the capacity of link $j$ is $C_{j}$.

2) A call on route $r$ uses $A_{j r}$ circuits from link $j, A_{j r} \in$ $\{0,1\}$. Let $\mathbf{R}$ be the set of all possible routes.

3) The calls requesting route $r$ arrive as an independent Poisson process of rate $\lambda_{r}$.

4) The duration of each call is exponentially distributed with unit mean and independent of earlier arrival and holding times.

5) A link $j, j=1,2, \ldots, J$ is absorbed at time $t$ if a call requesting route $r$ arrives at time $t$ but there are no circuits free in link $j$ provided $A_{j r}=1$.

Let $n_{r}$ be the number of calls in progress on route $r$ and define $\mathbf{n}=\left(n_{r}, r \in \mathbf{R}\right)$ and $\mathbf{C}=\left(C_{j}, j=1,2, \ldots, J\right)$. Now considering an $|\mathbf{R}|$-dimensional state space with a constraint An $\leq \mathbf{C}$, an explicit form might be thought to provide the complete solution of absorption probabilities. However, the number of routes and thus the size of the state space grows rapidly with the number of links. The size of state space also increases with the capacities of the links $C_{1}, C_{2}, \ldots, C_{J}$. So, it is impractical to compute the explicit forms of absorption probabilities except for some small networks.

The assumption that all the $J$ links are independent will yield an upper bound on absorption probability for each link. The absorption probability of the $j$-th link at time $t, P_{\text {abs }}^{j}(t)$ is bounded by the following function:

$$
P_{\text {abs }}^{j}(t) \leq 1-f\left(\sum_{r \in \mathbf{R}} A_{j r} \lambda_{r}, C_{j}, t\right) \quad j=1,2, \ldots, J .
$$

The function $f$ is the same as defined in Section V.B. But this may lead to a very conservative upper bound because the links may be far from being independent in reality. The links can still be assumed independent if the arrival rates are thinned properly for each link, and this results in a good approximation of the absorption probabilities. 
In this section, we will describe a method that will help to thin the arrival rates in a proper way and which approximates the absorption probabilities as a function of time. Motivated by the Erlang fixed point approximation in steady state condition [11], the carried traffic on link $j$ at time $t$ is approximated by a Poisson process with arrival rate $\sum_{r} A_{j r} \lambda_{r} \prod_{i \neq j}\left(1-P_{\text {abs }}^{i}(t)\right)^{A_{i r}}$ when $P_{\text {abs }}^{i}(t)$ is the absorption probability of link $i$ at time $t$. It is assumed that when $A_{j r}=1$ the stream of rate $\lambda_{r}$ is thinned by a factor $1-P_{\text {abs }}^{i}(t)$ at each link $i \in r-\{j\}$ before being offered to link $j$ and the thinnings are independent from link to link and over all routes containing the link $j$. Under this assumption, the carried traffic on link $j$ is a Poisson process with time-varying arrival rate $\sum_{r} A_{j r} \lambda_{r} \prod_{i \neq j}\left(1-P_{\text {abs }}^{i}(t)\right)^{A_{i r}}$.

The absorption probability of a link $j, j=1,2, \ldots, J$ can be calculated from the following time-dependent nonlinear equations:

$$
P_{\text {abs }}^{j}(t)=F\left(\sum_{r \in \mathbf{R}} A_{j r} \lambda_{r} \prod_{i \in r-\{j\}}\left(1-P_{\text {abs }}^{i}(t)\right), C_{j}\right)
$$

where $F(\lambda(t), C)$ is the absorption probability of a single link of capacity $C$ with time-dependent Poisson arrival rate $\lambda(t)$ and mean service time unity at time $t$.

The probability that a route $r \in \mathbf{R}$ is absorbed at time $t$ is

$$
L_{r}(t)=1-\prod_{j \in r}\left(1-P_{\text {abs }}^{j}(t)\right) .
$$

In steady state and under a limiting regime where the offered traffic and capacities are scaled proportionally, a simple approximation of blocking probability converges to the correct value and the approximation is more and more accurate as the capacity of the network increases [11]. Our results for a transient state condition also indicate that the approximation of absorption probability at any time $t$ will be more accurate for larger capacities in a network.

\section{RESULTS AND DISCUSSION}

In this section, we present the results of the approximation technique for large networks and compare it with the simulations to study its performance. Experiments are performed for various topologies under different traffic conditions with different capacities of links.

We consider two different topologies, a 9-node ring and a 20-node network representing a skeleton of the original Arpanet. For network traffic, we assumed fixed routing and here we consider the shortest path routing for our experiments. Again we assume identical traffic arrival rates in all the routes with equal hop lengths and the traffic in routes with different hop lengths are varying according to the following:

$$
\lambda_{H}=q^{H-1} \cdot \lambda_{1}
$$

where $\lambda_{H}$ is the arrival rate on an $H$-hop route; $q=1$ for uniform traffic; $q<1$ if traffic is dominated by smaller hop routes; and $q>1$ if traffic dominated by larger hop routes.

\section{A. Performance for Ring Network}

Here we consider a 9-node ring which is a regular network with an odd number of links. So the routes under shortest path

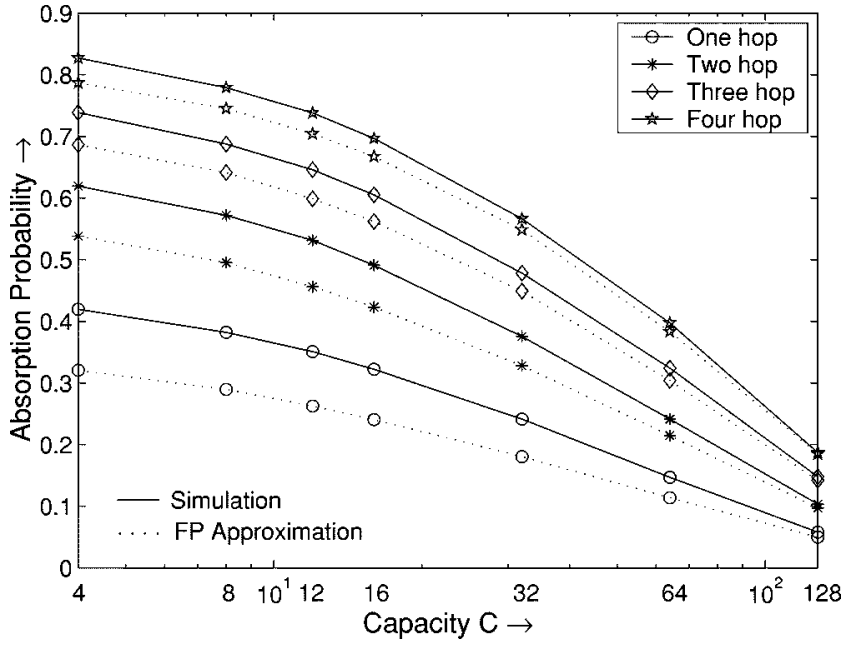

Fig. 6. Absorption probability in a nine-node ring network under nonuniform traffic pattern $(q=0.5)$ at $t=1.5$. Note that the approximation converges for larger capacity.

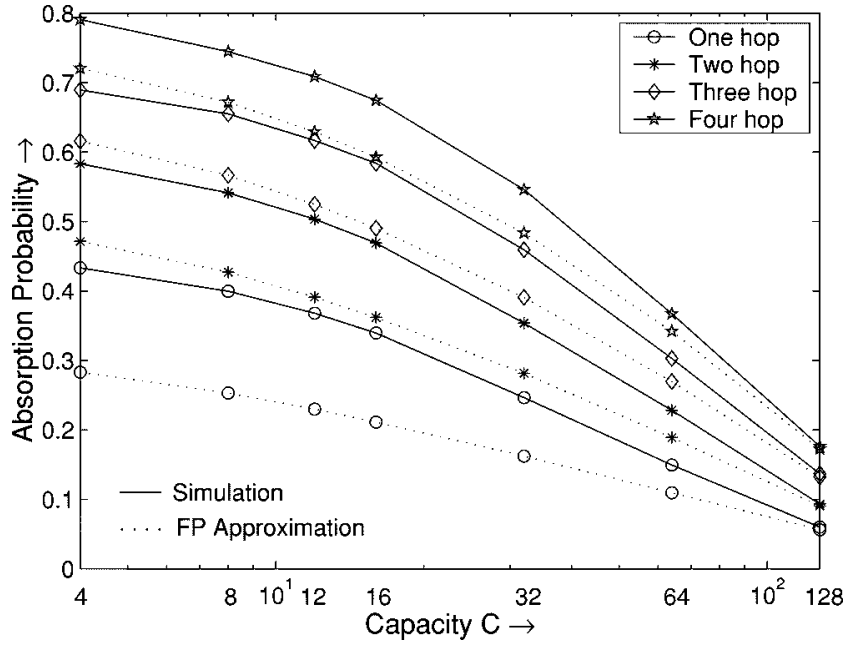

Fig. 7. Absorption probability in a nine-node ring network under uniform traffic pattern $(q=1)$ at $t=1.5$.

routing are unique. For the 9-node ring network, the maximum hop length is 4 and the total number of possible routes is 36,9 each with hop lengths ranging from 1 to 4 .

The absorption probabilities for single hop routes are approximated by the solution of a set of nonlinear coupled equations as shown in (15) with $J=9,|\mathbf{R}|=36$ and identical link capacities $C_{i}=C$. The absorption probabilities for multihop routes are approximated based on the individual link absorption probabilities as shown in (16). The simulation and the approximation are compared for seven different capacities, $C=$ $4,8,12,16,32,64$ and 128 , under two different traffic patterns, $q=0.5$ and $q=1$, with $\lambda_{1}=0.35 C$ and $0.12 C$, respectively.

For both the simulation and approximation, the absorption probabilities for one-, two-, three-, and four-hop routes are averaged for $t=1.5$ and the average absorption probabilities are plotted for different values of capacities in Figs. 6 and 7 for nonuniform $(q=0.5)$ and uniform traffic, respectively.

Observe that the approximation is good for large values of $C$ but not for small values of $C$. For small values of $C$, the approximation is better in the multihop case than in the single hop case. To see why this is so, observe that for the value of $t$ considered 


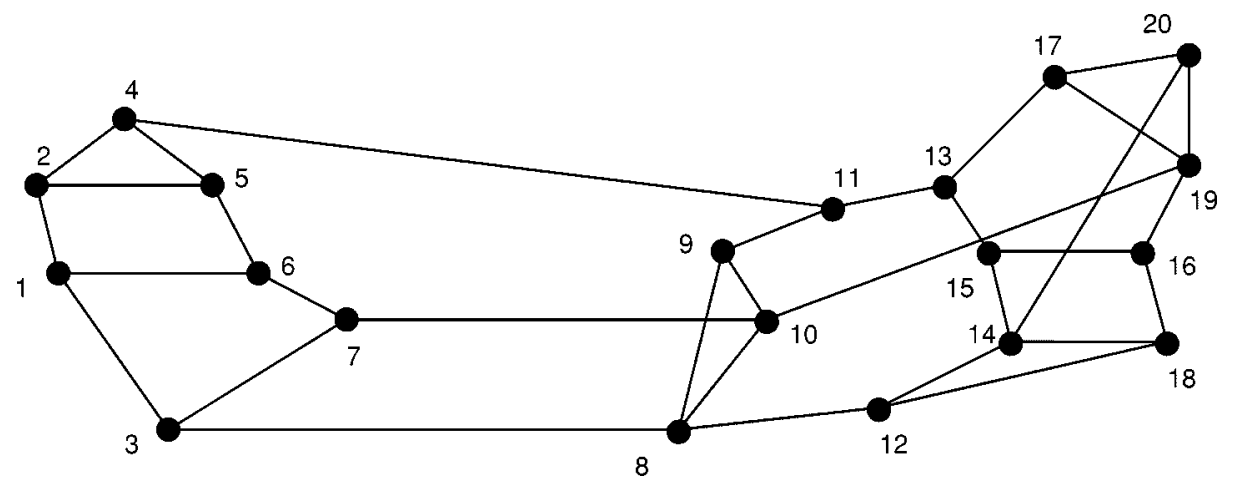

Fig. 8. 8 A 20-node 32-link network representing a skeleton of the original Arpanet.

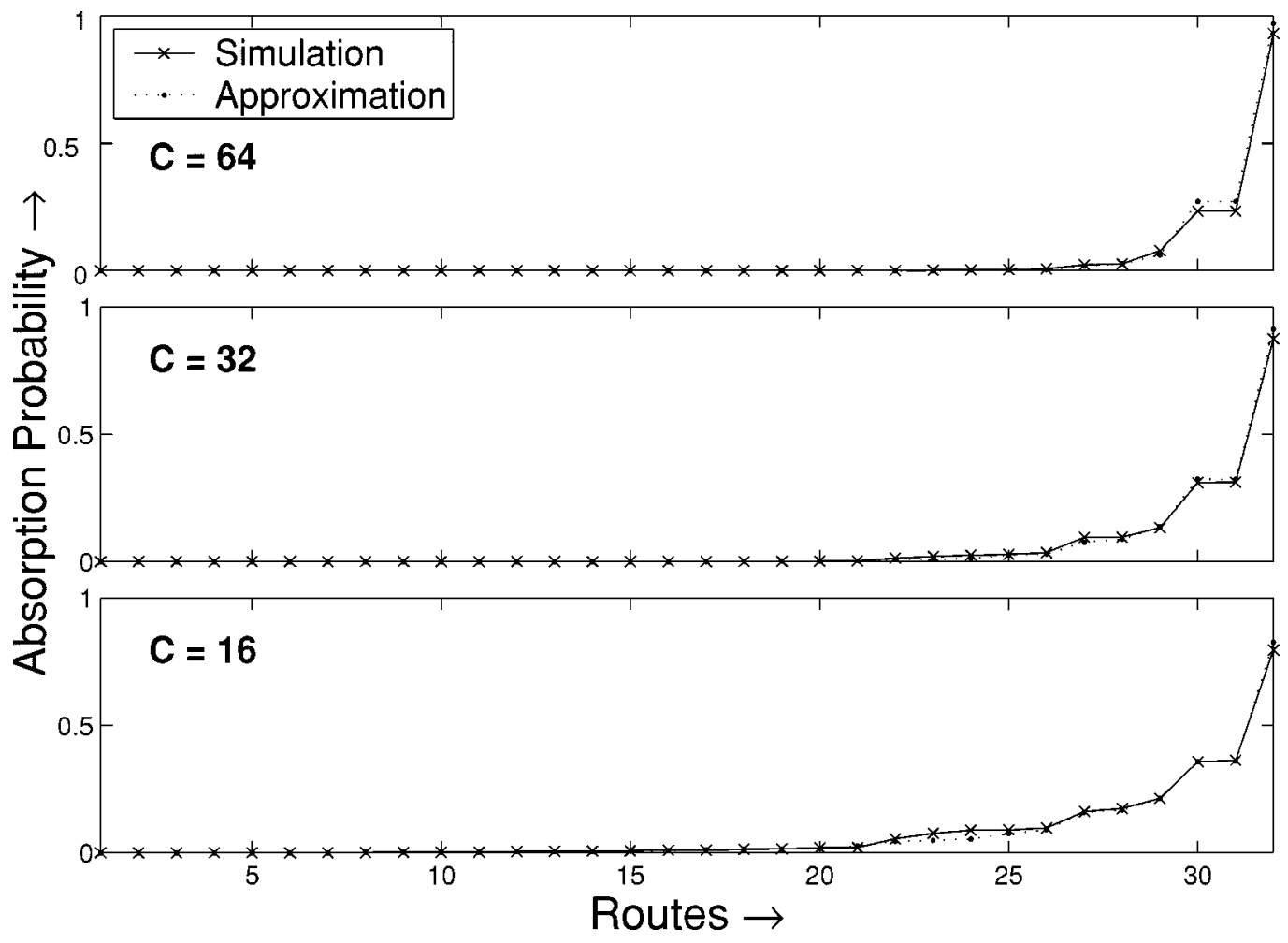

Fig. 9. Absorption probability for single-hop routes in the 20-node ARPA network at $t=1$.

in Figs. 6 and $7(t=1.5)$, the FP approximation is an underestimate. The independence assumption when applied to the exact one-hop probabilities would result in an overestimate for multihop probabilities, as we saw in the two-link case. Since we are applying the independence assumption to an underestimate of the single link probabilities, there is a degree of cancellation in the errors due to the two-step approximation (FP followed by independence) resulting in a better approximation for the multihop case.

Observe that the approximate absorption probability converges for larger values of capacity under both uniform and nonuniform traffic patterns. This result is useful for designing a ring with low absorption probability.

\section{B. Performance for ARPA Network}

We consider the 20-node ARPA network shown in Fig. 8 as an example of an arbitrary and moderately large topology to study the performance for arbitrary networks. Routes are selected as the shortest paths between any two nodes. For a 20-node network, the total number of possible routes is 190 , provided there is only one route between any two nodes. Under shortest path routing, the maximum possible hop length is 6 and the number of routes with $1,2,3,4,5$, and 6 hops are $32,52,56,32,17$, and 1 , respectively. Here we consider only the nonuniform traffic with $q=0.5$ and the absorption probabilities are calculated for different values of $C$ with $\lambda_{1}=0.2 C$.

For single hop routes, the approximate absorption probabilities are calculated from (15) with $J=32$ and $|\mathbf{R}|=190$. For multihop routes, these are obtained from (16) using the appropriate link absorption probabilities. For all the routes with equal hop length, the absorption probabilities obtained from the simulation and that calculated from the approximation are sorted in ascending order for a particular value of time and the resulting absorption probabilities are plotted in Figs. 9-12 for one-, two-, three-, and four-hop routes, respectively. 


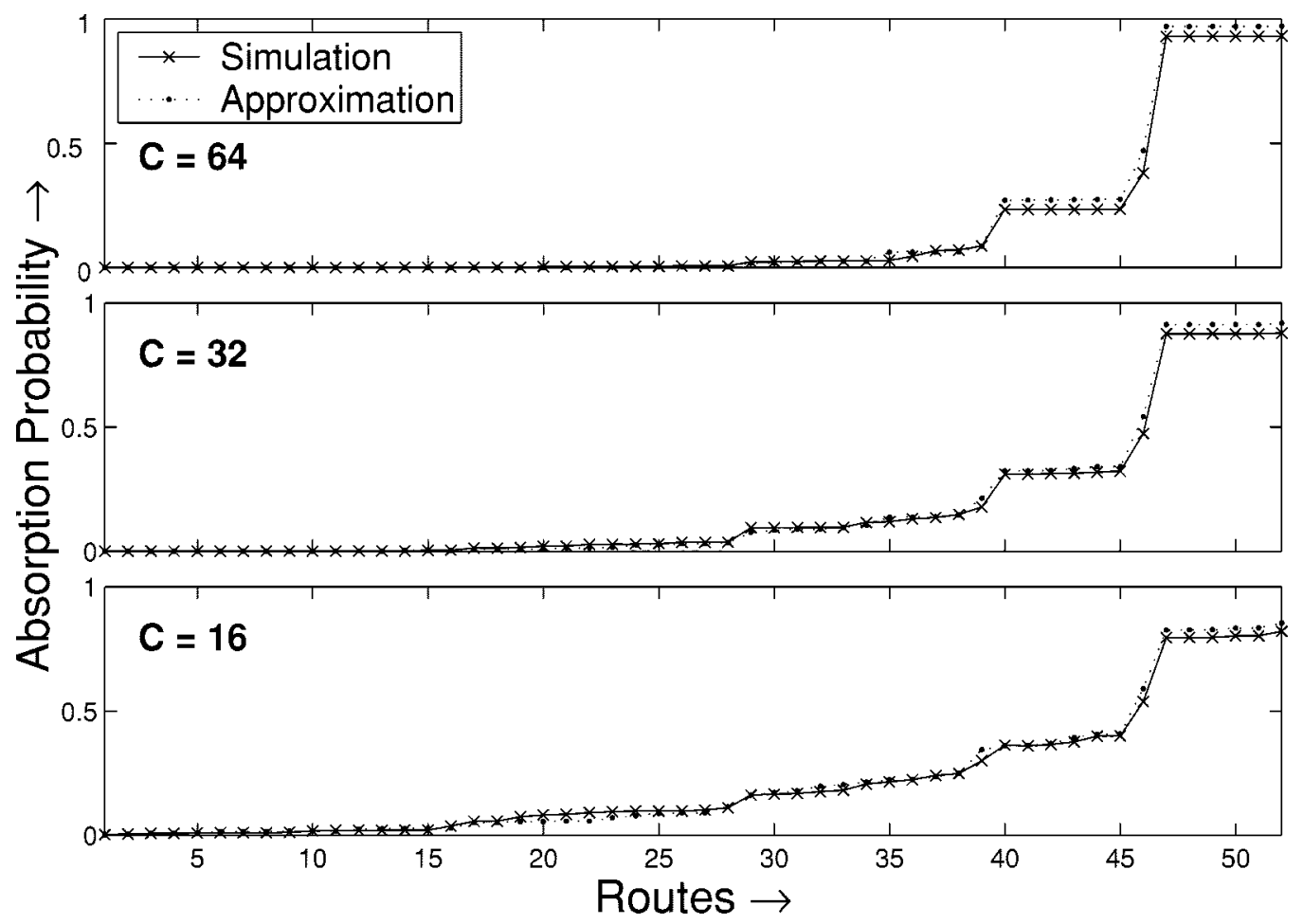

Fig. 10. Absorption probability for two-hop routes in the 20-node ARPA network at $t=1$.

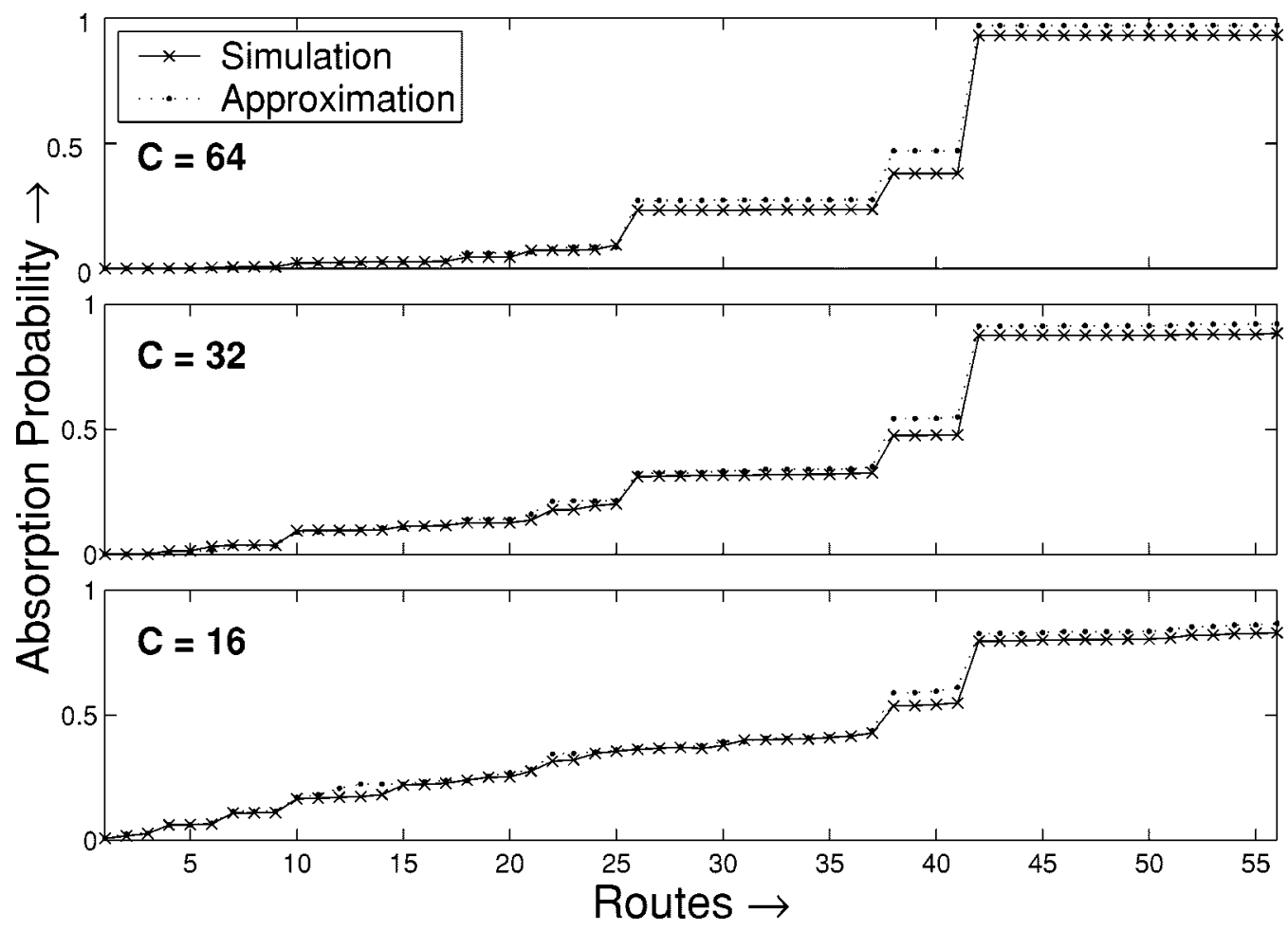

Fig. 11. Absorption probability for three-hop routes in the 20-node ARPA network at $t=1$.

Experiments are performed for various values of capacity with $\lambda_{1}=0.2 C$ and the absorption probabilities are calculated for $t=0$ to $t=3$ with a time increment 0.001 . We present the times taken for computation at different values of capacity in Table I. All computations were done on a Sun Ultra SPARC system running at $450 \mathrm{MHz}$. Note that the computation times are on the order of minutes.
It is observed from Fig. 9 that the absorption probabilities for different links are varying widely. This happens because the connections in the network are highly irregular and the number of routes through a link varies from 3 to 43 . There are some links e.g., $(10,19),(11,13),(4,11),(7,10)$, and $(3,8)$, through which the number of routes is very large and this results in very high absorption probabilities for those links. There are other 


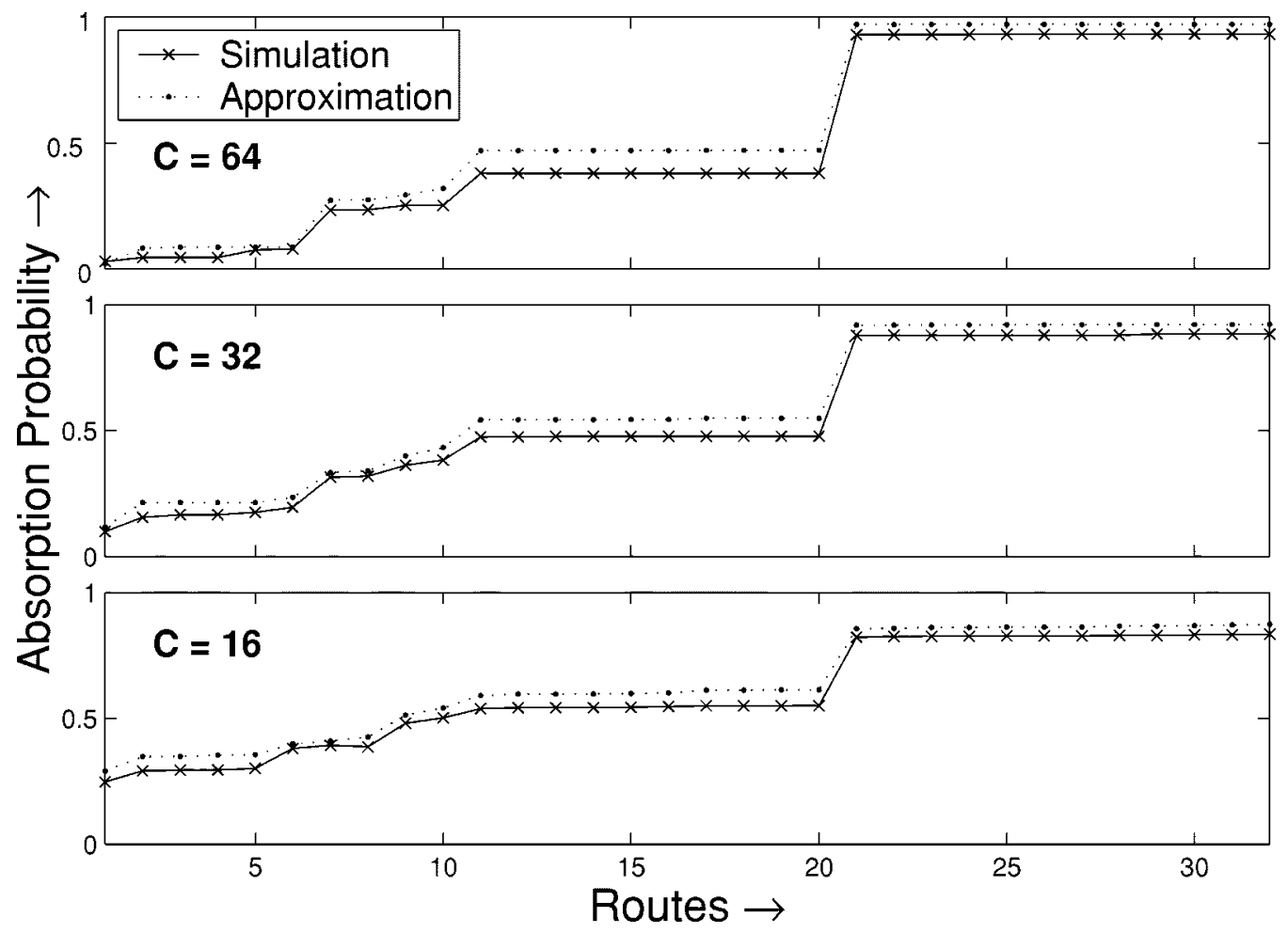

Fig. 12. Absorption probability for four-hop routes in the 20-node ARPA network at $t=1$.

TABLE I

TIMES FOR COMPUTATION AT DIFFERENT VALUES OF CAPACITY

\begin{tabular}{c|c||c|c}
\hline \hline $\begin{array}{c}\text { Capacity } \\
\mathrm{C}\end{array}$ & $\begin{array}{c}\text { Time } \\
\min : \text { sec }\end{array}$ & $\begin{array}{c}\text { Capacity } \\
\mathrm{C}\end{array}$ & $\begin{array}{c}\text { Time } \\
\min : \mathrm{sec}\end{array}$ \\
\hline \hline 16 & $0: 35$ & 80 & $2: 31$ \\
\hline 32 & $0: 42$ & 96 & $3: 23$ \\
\hline 48 & $1: 07$ & 112 & $4: 42$ \\
\hline 64 & $1: 47$ & 128 & $6: 27$ \\
\hline
\end{tabular}

links like $(1,6),(2,5),(3,7)$, and $(14,18)$ which are used less often and this results in very low absorption probabilities for those links. So with equal capacity some of the links are overdimensioned and the situation becomes progressively worse as the capacity increases. So what we need is the proper dimensioning of the network so that the absorption probability of any route be less than a threshold value at the time of interest.

From Figs. 9-12 we observe that the absorption probability for each individual link as well as for each individual route is well approximated by the method we have presented in this paper and from Table I we observe that the method is also very efficient.

Here we present the capacities alloted to various links of the ARPA network based on both the blocking and absorption probabilities and compare the results. For all the computations we assumed $\lambda_{1}=12$. First the capacities are designed so that the blocking probabilities of all the 190 routes are less than $0.01(1 \%)$. We consider two target absorption probabilities, $1 \%$ and $10 \%$, because it may be appropriate to dimension the network for a higher value of absorption probability, say $10 \%$, than the target blocking probability, say $1 \%$. We then solve the dimensioning problem by iterating on the analysis problem so that the absorption probabilities of all the routes are less than $0.01(1 \%)$ and $0.1(10 \%)$ at time $t=0.5,1,1.5$, and 2 . The results are presented in Table II.

It is observed from Table II that the capacity allocation based on the absorption probability is much better than that based on the blocking probability if the network capacities are upgraded within a time interval equal to or less than twice the mean connection holding time.

For an illustration, let us assume the mean holding time of a lightpath is one year. Allot the capacities based on the absorption probability as shown in Fig. 13. The absorption probabilities for routes with different hops are plotted in Fig. 14. Note that all the absorption probabilities are less than 0.1 at $t=0.5$ with $\lambda_{1}=12$. This means that the operator has a $90 \%$ or better chance of not having to upgrade the network for a time period of six months. Observe that the capacities required are only $42 \%$ of that required using the blocking probability model.

\section{CONCLUSION}

We have introduced the concept of absorption probability for dimensioning optical networks with long-lived lightpaths. We have proposed a method to approximate the absorption probabilities of wavelength-routed networks with arbitrary topologies and traffic patterns. We have shown that the approximations are close to the simulation results and computationally this method is efficient. Thus, a large network can be dimensioned so that the absorption probability of any route is less than the threshold value at the time of interest.

Here we considered only fixed routing. Apart from dimensioning, routing can be based on absorption probability, that is, for a given network with arbitrary topology and traffic pattern, 
TABLE II

CAPaCities Alloted to DifFerent LinKS OF THE ARPA NETWORK

\begin{tabular}{|c|c|c|c|c|c|c|c|c|c|}
\hline \multirow{3}{*}{$\begin{array}{c}\text { Link } \\
\text { No }\end{array}$} & \multirow{3}{*}{\begin{tabular}{|c|} 
Bl \\
Prob \\
0.01 \\
\end{tabular}} & \multicolumn{8}{|c|}{ Absorption Probability } \\
\hline & & \multicolumn{2}{|c|}{$\mathrm{t}=0.5$} & \multicolumn{2}{|c|}{$\mathrm{t}=1$} & \multicolumn{2}{|c|}{$\mathrm{t}=1.5$} & \multicolumn{2}{|c|}{$t=2$} \\
\hline & & 0.1 & 0.01 & 0.1 & 0.01 & 0.1 & 0.01 & 0.1 & 0.01 \\
\hline$\overline{\overline{1}}$ & $\overline{\overline{53}}$ & $\overline{23}$ & $\overline{27}$ & $\overline{34}$ & $\overline{39}$ & $\overline{41}$ & $\overline{46}$ & 46 & $\overline{51}$ \\
\hline 2 & 68 & 29 & $\overline{33}$ & 44 & 48 & 53 & 58 & 59 & 65 \\
\hline 3 & 38 & 16 & 19 & 23 & 28 & 28 & 33 & 31 & 36 \\
\hline 4 & $\overline{62}$ & 27 & $\overline{31}$ & 40 & 46 & $\overline{49}$ & 54 & 54 & 60 \\
\hline 5 & 34 & 14 & 17 & 20 & 25 & 25 & 29 & 28 & 32 \\
\hline 6 & 34 & $\overline{14}$ & 17 & 20 & 25 & 25 & 29 & 28 & 32 \\
\hline 7 & 100 & 42 & 48 & 64 & 71 & 78 & 85 & 86 & 94 \\
\hline 8 & 58 & 25 & 29 & 37 & 42 & 46 & 50 & 50 & 56 \\
\hline 9 & 113 & 47 & 53 & 73 & 79 & 88 & 96 & 98 & 106 \\
\hline 10 & 78 & 33 & 38 & 50 & 56 & 61 & 67 & 68 & 74 \\
\hline 11 & 94 & 40 & 45 & 60 & 66 & 73 & 79 & 81 & 88 \\
\hline 12 & 107 & 45 & 50 & 69 & 75 & 83 & 90 & 91 & 99 \\
\hline 13 & 74 & 31 & 36 & 46 & 52 & 56 & 62 & 63 & 69 \\
\hline 14 & $\overline{90}$ & 38 & 43 & 58 & 64 & 70 & 77 & 77 & 85 \\
\hline 15 & 101 & 42 & 47 & 64 & 71 & 78 & 85 & 86 & 94 \\
\hline 16 & 68 & 29 & 33 & 42 & 49 & 52 & 58 & 58 & 64 \\
\hline 17 & 90 & 37 & 42 & 57 & 63 & 68 & 75 & 76 & 83 \\
\hline 18 & 147 & 61 & 67 & 94 & 101 & 113 & 122 & 126 & 135 \\
\hline 19 & 113 & 48 & 53 & 73 & 79 & 88 & 95 & 98 & 106 \\
\hline 20 & 48 & 20 & 24 & 30 & 35 & 36 & 41 & 40 & 45 \\
\hline 21 & 70 & 30 & 34 & 45 & 51 & 54 & 60 & 60 & 66 \\
\hline 22 & 86 & 36 & $\overline{41}$ & 54 & 61 & 66 & 73 & 73 & 80 \\
\hline 23 & 78 & 32 & 37 & 49 & 55 & 59 & 66 & 66 & 72 \\
\hline 24 & 62 & 26 & 31 & 40 & 45 & 48 & 54 & 53 & 59 \\
\hline 25 & 38 & 16 & 19 & 23 & 28 & 28 & 33 & 31 & 36 \\
\hline 26 & 72 & 31 & 35 & 47 & 52 & 57 & 63 & 63 & 69 \\
\hline 27 & 64 & 27 & 31 & 41 & 46 & 49 & 55 & 54 & 60 \\
\hline 28 & 68 & 29 & 34 & 44 & 50 & 54 & 60 & 60 & 66 \\
\hline 29 & 92 & 39 & 44 & 59 & 65 & 71 & 78 & 79 & 86 \\
\hline 30 & 48 & 20 & 24 & 30 & 35 & 37 & 41 & 40 & 46 \\
\hline 31 & 74 & 32 & 36 & 48 & 54 & 58 & 65 & 65 & 71 \\
\hline 32 & 70 & 30 & 35 & 45 & 51 & 54 & 60 & 60 & $\overline{66}$ \\
\hline
\end{tabular}

\begin{tabular}{l||c|c|c|c|c|c|c|c|c}
\hline \hline Tot & 2392 & 1009 & 1153 & 1523 & 1707 & 1846 & 2039 & 2048 & 2251 \\
\hline
\end{tabular}

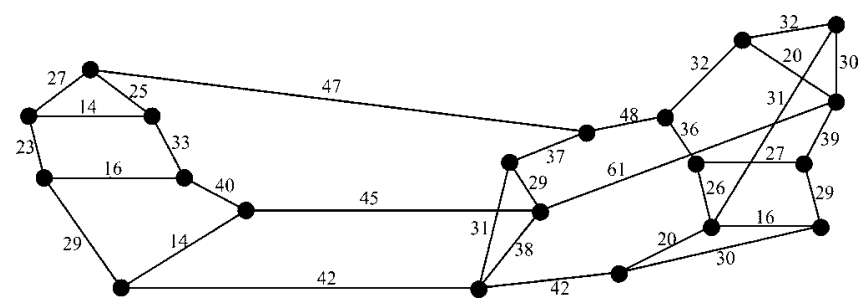

Fig. 13. Capacities alloted to different links of the ARPA network for $t=0.5$.

the routes can be selected in such a way so that the absorption probability of any route is low at the time of interest. This is a topic for further research.

\section{APPENDIX A}

Solving the system of (5), we get

$$
\mathbf{P}(t)=e^{\mathbf{A} t} \mathbf{P}(0) \text {. }
$$

Let $\mathbf{B}=e^{\mathbf{A} t}=\left[\mathbf{b}_{0} \mathbf{b}_{1} \ldots \mathbf{b}_{K+1}\right]$ where $\mathbf{b}_{i}$ s are the column vectors of $\mathbf{B}$

$$
\Rightarrow \mathbf{P}(t)=\mathbf{b}_{0} \quad \text { and } \quad P_{K+1}(t)=B_{K+1,0}=b_{0, K+1} .
$$

Theorem 1:

1) All the $(K+2)$ eigenvalues of $\mathbf{A}$ are real and distinct.

2) One eigenvalue of $\mathbf{A}$ is zero and all other eigenvalues are negative.

Let us assume $\alpha_{0}, \alpha_{1}, \ldots \alpha_{K+1}$ are the $(K+2)$ eigenvalues of $\mathbf{A}$ arranged in decreasing order. From Theorem 1 it follows that all the eigenvalues are distinct. So from Sylvester's theorem [17] we get

$$
\mathbf{B}=e^{\mathbf{A} t}=\sum_{r=0}^{K+1} e^{\alpha_{r} t} \frac{\left[\mathrm{D}\left(\alpha_{r}\right)\right]}{\Delta^{\prime}\left(\alpha_{r}\right)}
$$

where $[\mathbf{D}(\alpha)]=$ adjoint matrix of $[\alpha \mathbf{I}-\mathbf{A}]$ and

$$
\begin{aligned}
\Delta^{\prime}\left(\alpha_{r}\right) & =\prod_{i \neq r}\left(\alpha_{r}-\alpha_{i}\right) \\
{\left[\mathrm{D}\left(\alpha_{r}\right)\right]_{K+1,0} } & =(-1)^{K+1} \cdot \text { Minor } M_{0, K+1} \text { of }\left[\alpha_{r} \mathbf{I}-\mathbf{A}\right] \\
& =\lambda_{0} \lambda_{1} \ldots \lambda_{K}=c(\text { say }) \\
\text { So } P_{K+1}(t) & =\sum_{r=0}^{K+1} \frac{e^{\alpha_{r} t}}{\Delta^{\prime}\left(\alpha_{r}\right)} \cdot\left[\mathrm{D}\left(\alpha_{r}\right)\right]_{K+1,0} \\
& =c \cdot \sum_{r=0}^{K+1} \frac{e^{\alpha_{r} t}}{\Delta^{\prime}\left(\alpha_{r}\right)} .
\end{aligned}
$$

Now, one of the $(K+2)$ eigenvalues of $\mathbf{A}$ is zero and all others are negative, so $\alpha_{0}=0$.

Define a matrix $\mathbf{M}$ removing the last row and last column of matrix $\mathbf{A}$. Thus, $\alpha_{1}, \alpha_{2}, \ldots \alpha_{K+1}$ are the eigenvalues of $\mathbf{M}$. (Shown in (21) at the bottom of the next page.)

\section{A. Property of the Coefficients}

1) The coefficients $a_{1}, a_{2}, \ldots a_{K+1}$ are alternating in sign with $a_{1}$ positive.

2)

$$
P_{K+1}(0)=0 \Rightarrow \sum_{r=1}^{K+1} a_{r}=1 .
$$

If $\alpha_{1}$ is very close to zero, from (20)

$$
\Delta^{\prime}\left(\alpha_{1}\right)=\alpha_{1} \prod_{i=2}^{K+1}\left(\alpha_{1}-\alpha_{i}\right) \approx(-1)^{K} \prod_{i=1}^{K+1} \alpha_{i}=-c .
$$

So from (21), $a_{1}=-c / \Delta^{\prime}\left(\alpha_{1}\right) \approx 1$ and from (22), $a_{i} \approx 0$ for $i>1$.

\section{B. Bound on the Dominant Eigenvalue}

Theorem 2: The dominant eigenvalue of $\mathbf{M}$ must be greater than the negative of the minimum arrival rate, i.e.,

$$
\alpha_{1} \geq-\min _{0 \leq i \leq K} \lambda_{i}
$$

Theorem 3: If $K$ increases, the dominant eigenvalue also increases.

Theorem 4: If $\lambda_{n}=\lambda$ and $\mu_{n}=n \mu, 1 \leq n \leq K, \rho=\lambda / \mu$, the dominant eigenvalue of $\mathbf{M}$ lies between $-\mu$ and 0 iff $\rho \leq$ $K+1$ and lies between $-2 \mu$ and 0 iff $\rho \leq K+1+\sqrt{K+1}$. 
Theorem 5: For the matrix $\mathbf{M}$ with $\lambda_{n}=\lambda$ and $\mu_{n}=n \mu$, the eigenvalues decrease monotonically with the increase of $\rho$ and the difference between any two consecutive eigenvalues is greater than $\mu$, i.e.,

$$
\alpha_{i-1}-\alpha_{i} \geq \mu \text { for all } i, 1<i \leq K+1 \text {. }
$$

Equality holds for $\rho=0$ only.

\section{Computer Experiments}

In Fig. 15, all the $(K+1)$ normalized eigenvalues $\left(\alpha_{i} / \mu\right)$ are plotted against $\rho$ for $K=20, i=1,2, \ldots, 21$ and we can observe the following.

1) $\left(\alpha_{i} / \mu\right)$ is monotonically decreasing with $\rho$ for all $i$.

2) The difference between any two consecutive normalized eigenvalues $\left(\left(\alpha_{i} / \mu\right)-\left(\alpha_{i-1} / \mu\right)\right)$ is monotonically increasing with $\rho$ and the difference is minimum for $\rho=0$ for all $i, 1<i \leq K+1$ and the minimum difference is 1 .

\section{APPENDIX B}

\section{A. Computation of Exact Absorption Probability for a Two-Link Network}

Here we introduce a 3-D Markov chain model to calculate the exact absorption probability for a two-link network. We consider the more general case: different servicetimes for different type of calls. Here we state the assumptions about the Markov chain model.

\section{B. Assumptions}

1) Departure of calls are Poisson with mean service time $1 / \mu_{i}$ for a call of type- $i, i=1,2,3$.

2) A state of the Markov chain is represented by $\left(n_{1}, n_{2}, n_{3}\right)$ where $n_{i}$ is the number of type- $i$ calls in progress simultaneously.

3) Change of state takes place due to an arrival or departure of a call that takes place one at a time.

The variables $n_{1}, n_{2}$ and $n_{3}$ represent the three axes of the 3-D Markov state space. From any state of the Markov chain, a maximum of six transitions are possible, two in each direction, one for an arrival, and one for a departure. For a general state $\left(n_{1}, n_{2}, n_{3}\right)$, all the transition states and the transition rates are shown in Fig. 16.
It is assumed that the transition starts from the initial state $(0,0,0)$ at time $t=0$ and the process progresses according to the arrivals and departures of the lightpaths and finally the system reaches an absorbing state. A state can be classified in the following way.

1) $\left(n_{1}, n_{2}, n_{3}\right)$ is a transient state if $0 \leq n_{1}+n_{3} \leq K_{1}$ and $0 \leq n_{2}+n_{3} \leq K_{2}$.

2) ( $\left.n_{1}, n_{2}, n_{3}\right)$ is an absorbing state if $n_{1}+n_{3}=K_{1}+1$ or $n_{2}+n_{3}=K_{2}+1$.

So with the above constraints, the 3-D Markov state space will be a polyhedron bounded by four triangular faces and one rectangular face.

The total number of transient states is

$$
N=\left(K_{1}+1\right)\left(K_{2}+1\right)+K_{1} K_{2}+\ldots+\left(\left|K_{1}-K_{2}\right|+1\right) \text {. }
$$

If $K_{1}=K_{2}=K$, this reduces to $((K+1)(K+2)(2 K+$ $3)) /(6)$.

The total number of absorbing states is $=\left(K_{1}+2\right)\left(K_{2}+\right.$ 2 ) -1 (considering $\left(K_{1}+1, K_{2}+1,0\right)$ is not an absorbing state).

Let us assume $P_{n_{1}, n_{2}, n_{3}}(t)$ is the probability of finding the system in state $\left(n_{1}, n_{2}, n_{3}\right)$ at time $t$.

We can write the state transition equations as follows:

$$
\begin{aligned}
P_{n_{1}, n_{2}, n_{3}}^{\prime}(t)= & -\left(\lambda_{1}+n_{1} \mu_{1}\right) P_{n_{1}, n_{2}, n_{3}}(t) \\
& +f\left(n_{1}\right) \lambda_{1} P_{n_{1}-1, n_{2}, n_{3}}(t) \\
& +g\left(n_{1}+1, n_{2}, n_{3}\right)\left(n_{1}+1\right) \\
& \times \mu_{1} P_{n_{1}+1, n_{2}, n_{3}}(t) \\
& -\left(\lambda_{2}+n_{2} \mu_{2}\right) P_{n_{1}, n_{2}, n_{3}}(t) \\
& +f\left(n_{2}\right) \lambda_{2} P_{n_{1}, n_{2}-1, n_{3}}(t) \\
& +g\left(n_{1}, n_{2}+1, n_{3}\right)\left(n_{2}+1\right) \mu_{2} \\
& \times P_{n_{1}, n_{2}+1, n_{3}}(t) \\
& -\left(\lambda_{3}+n_{3} \mu_{3}\right) P_{n_{1}, n_{2}, n_{3}}(t) \\
& +f\left(n_{3}\right) \lambda_{3} P_{n_{1}, n_{2}, n_{3}-1}(t) \\
& +g\left(n_{1}, n_{2}, n_{3}+1\right)\left(n_{3}+1\right) \\
& \times \mu_{3} P_{n_{1}, n_{2}, n_{3}+1}(t)
\end{aligned}
$$

where

$$
f(n)=I_{\{n \geq 1\}}
$$

$$
\begin{aligned}
\mathbf{M} & =\left[\begin{array}{ccccc}
-\lambda_{0} & \mu_{1} & \ldots & 0 & 0 \\
\lambda_{0} & -\left(\lambda_{1}+\mu_{1}\right) & \ldots & 0 & 0 \\
\vdots & \vdots & & \vdots & \vdots \\
0 & 0 & \ldots & -\left(\lambda_{K-1}+\mu_{K-1}\right) & \mu_{K} \\
0 & 0 & \cdots & \lambda_{K-1} & -\left(\lambda_{K}+\mu_{K}\right)
\end{array}\right] \\
\Delta^{\prime}(0) & =(-1)^{K+1} \prod_{i=1}^{K+1} \alpha_{i}=(-1)^{K+1} \operatorname{det}[\mathbf{M}]=\lambda_{0} \lambda_{1} \ldots \lambda_{K}=c \\
P_{K+1}(t) & =c \cdot \sum_{r=0}^{K+1} \frac{e^{\alpha_{r} t}}{\Delta^{\prime}\left(\alpha_{r}\right)}=1+c \cdot \sum_{r=1}^{K+1} \frac{e^{\alpha_{r} t}}{\Delta^{\prime}\left(\alpha_{r}\right)}=1-\sum_{r=1}^{K+1} a_{r} \cdot e^{\alpha_{r} t} \quad \text { where } \quad a_{r}=-\frac{c}{\Delta^{\prime}\left(\alpha_{r}\right)}
\end{aligned}
$$



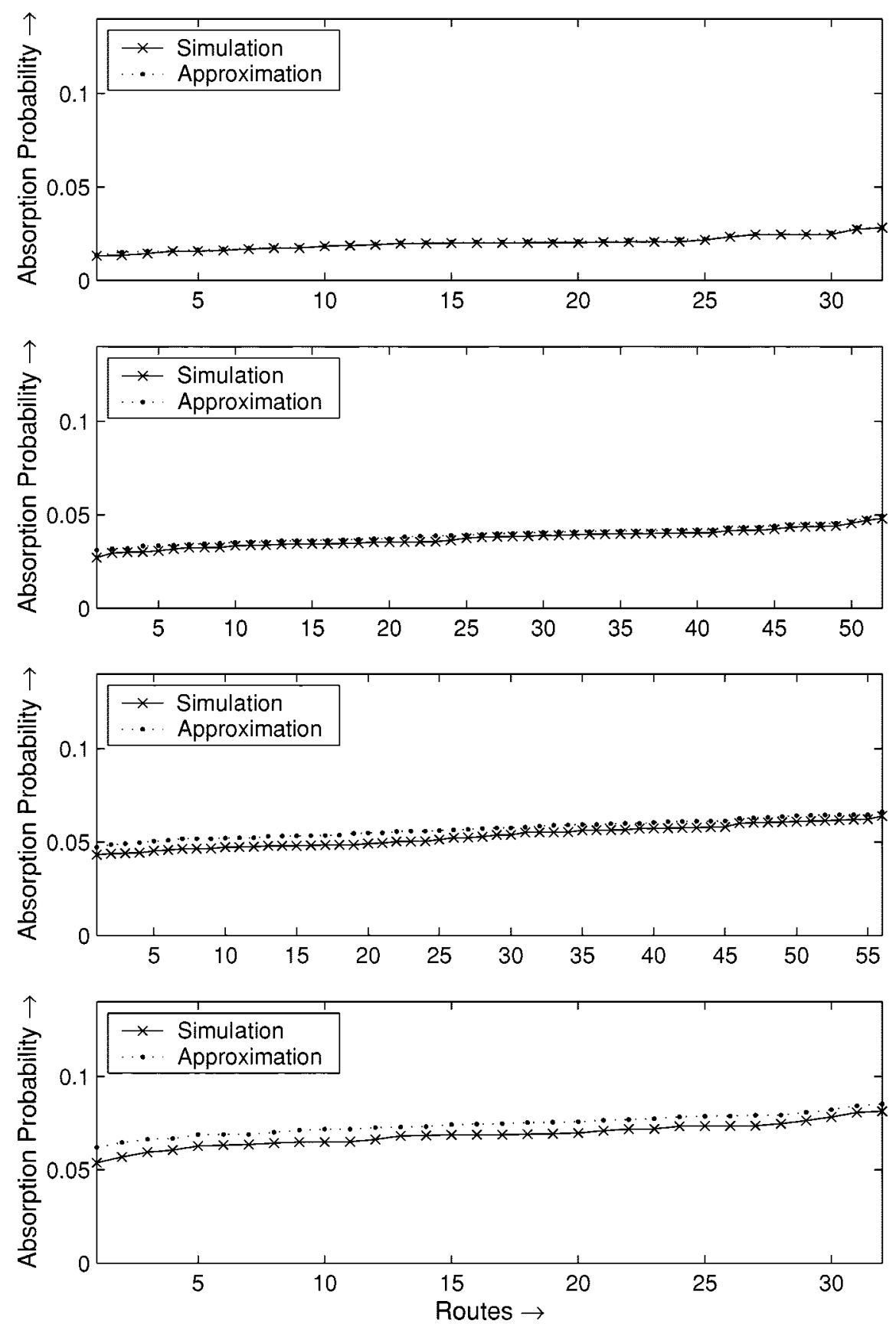

Fig. 14. Absorption probabilities at $t=0.5$ for one-hop, two-hop, three-hop, and four-hop routes in ARPA network with variable link capacities $\left(\lambda_{1}=12\right.$ and $q=0.5)$.

and

$$
g\left(n_{1}, n_{2}, n_{3}\right)=I_{\left\{n_{1}+n_{3} \leq K_{1} \& n_{2}+n_{3} \leq K_{2}\right\}} .
$$

The function $I$ is defined as

$$
I_{\text {statement }\}}= \begin{cases}1, & \text { if statement is true } \\ 0, & \text { otherwise. }\end{cases}
$$

For absorbing states

$$
\begin{aligned}
P_{n_{1}, n_{2}, n_{3}}^{\prime}(t)= & I_{\left\{n_{2}+n_{3} \leq K_{2}\right\}} P_{n_{1}-1, n_{2}, n_{3}}(t) \\
& +I_{\left\{n_{1}+n_{3} \leq K_{1}\right\}} \lambda_{2} P_{n_{1}, n_{2}-1, n_{3}}(t) \\
& +f\left(n_{3}\right) \lambda_{3} P_{n_{1}, n_{2}, n_{3}-1}(t)
\end{aligned}
$$

and initial condition:

$$
P_{n_{1}, n_{2}, n_{3}}(0)= \begin{cases}1, & \text { if } n_{1}, n_{2}, n_{3}=0 \\ 0, & \text { otherwise }\end{cases}
$$

We can observe that all the probabilities involved at the right-hand side of (24) are the probabilities of transient states only. So we can solve these from a set of differential equations. Let us define $\mathrm{P}(t)$ as a vector containing all the probabilities of the transient states and its length is $N$.

The system can be written in the following form:

$$
\mathbf{P}^{\prime}(t)=\mathbf{M P}(t)
$$




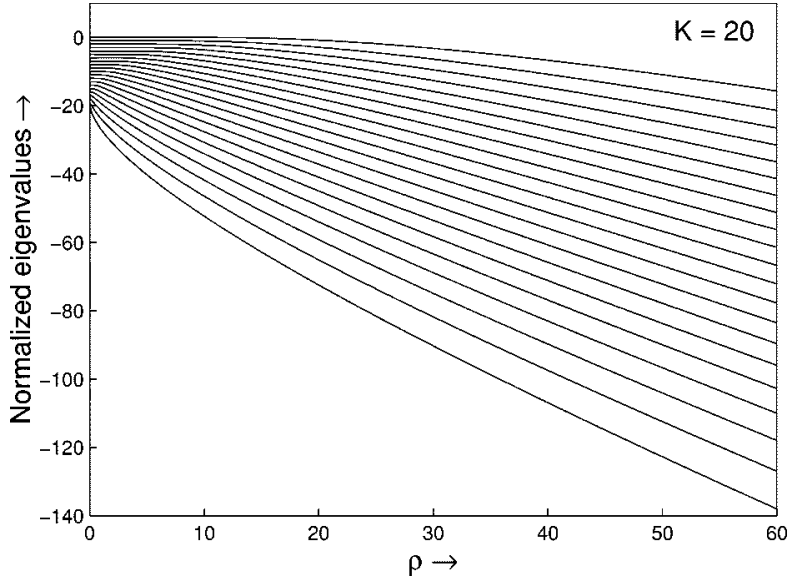

Fig. 15. Normalized eigenvalues versus $\rho$ for $K=20$.

where $\mathbf{M}$ is an $N \times N$ constant matrix. Solving, we get

$$
\mathbf{P}(t)=e^{\mathbf{M} t} \mathbf{P}(0)
$$

The absorption probability at time $t$

$$
\begin{aligned}
P_{\text {abs }}(t) & =\sum_{\substack{n_{1} \\
n_{1}+n_{3}=K_{1}+1 \\
\text { or } n_{2}+n_{3}=K_{2}+1}} P_{n_{1}, n_{2}, n_{3}}(t) \quad \text { for all } t>0 \\
& =1-\sum_{\substack{n_{1} \\
n_{1}+n_{3} \leq n_{1} \\
\& n_{2}+n_{3} \leq K_{2}}} \sum_{n_{3}} P_{n_{1}, n_{2}, n_{3}}(t) \quad \text { for all } t>0 .
\end{aligned}
$$

The exact absorption probability $P_{\text {abs }}(t)$ can be calculated easily from (29).

Although the simple explicit form seems easy to compute, it is far from the case. The dimension of the square matrix $\mathbf{M}$ is $N \times N$ and $N$ increases rapidly as capacity increases [see (23)]. So for large capacity, the memory requirement is huge and the solution requires a huge amount of computation which makes it impractical to compute $e^{\mathrm{M} t}$ directly.

In this section we present an efficient way to compute the absorption probability as a function of time

$$
\begin{aligned}
\mathbf{P}(t+h) & =e^{\mathbf{M}(t+h)} \mathbf{P}(0)=e^{\mathbf{M} h} e^{\mathbf{M} t} \mathbf{P}(0) \\
& =e^{\mathbf{M} h} \mathbf{P}(t) \\
\text { Again } \quad e^{\mathbf{M} h} & \approx \mathbf{I}+h \mathbf{M}+\frac{h^{2}}{2 !} \mathbf{M}^{2}
\end{aligned}
$$

The approximation of $e^{\mathrm{M} h}$ [shown in (31)] is very accurate for a sufficiently small $h$. This method requires very small amount of computation because it computes $e^{\mathrm{M} h}$ only once.

Again we can use the memory efficiently by storing only the nonzero elements of $\mathbf{M}$ because $\mathbf{M}$ has high sparsity and there is a nice symmetry around the main diagonal of $\mathbf{M}$ provided $\mathbf{P}(t)$ is chosen properly.

So starting from $\mathbf{P}(0), \mathbf{P}(t)$ and the absorption probability $P_{\text {abs }}(t)$, it can be computed for all values of $t=n h, n$ for a positive integer, and practically it will serve all our purposes.

\section{APPENDIX C}

\section{A. Approximation of Absorption Probability for a Two-Link Network}

Let $N_{i}$ be a random variable representing the number of type- $i$ calls in progress at time $t, i=1,2,3$ and let $K_{m}=\min \left(K_{1}, K_{2}\right) . N_{1}, N_{2}$ and $N_{3}$ can take the integer values from 0 to $K_{1}+1, K_{2}+1$ and $K_{m}+1$, respectively.

We can write the absorption probability in another way

$$
\begin{aligned}
P_{\mathrm{abs}}= & P\left[\left(N_{1}+N_{3}=K_{1}+1\right) \text { or }\left(N_{2}+N_{3}=K_{2}+1\right)\right] \\
= & \sum_{n_{3}=0}^{K_{m}+1} P\left(N_{3}=n_{3}\right) \\
& \cdot P\left[\left(N_{1}=K_{1}+1-n_{3}\right) \text { or }\left(N_{2}=K_{2}+1-n_{3}\right)\right] \\
P\left[\left(N_{1}=\right.\right. & \left.\left.K_{1}+1-n_{3}\right) \text { or }\left(N_{2}=K_{2}+1-n_{3}\right)\right] \\
= & 1-P\left[\left(N_{1} \leq K_{1}-n_{3}\right) \&\left(N_{2} \leq K_{2}-n_{3}\right)\right] \\
\approx & 1-P\left(N_{1} \leq K_{1}-n_{3}\right) \cdot P\left(N_{2} \leq K_{2}-n_{3}\right)
\end{aligned}
$$

where $P\left(N_{i} \leq K_{i}-n_{3}\right)$ is the no absorption probability of link- $i$ assuming it as an independent single link of capacity $\left(K_{i}-n_{3}\right), i=1,2$

$$
\begin{aligned}
P_{\text {abs }}(t) \approx & 1-\sum_{n_{3}=0}^{K_{m}} P_{\left(N_{3}=n_{3}\right)}(t) \\
& \cdot\left[P_{\text {noabs }}^{(1)}\left(K_{1}-n_{3}, t\right) \cdot P_{\text {noabs }}^{(2)}\left(K_{2}-n_{3}, t\right)\right] \\
= & 1-\sum_{n_{3}=0}^{K_{m}} P_{\left(N_{3}=n_{3}\right)}(t) \\
& \times\left[\sum_{i=1}^{K_{1}+1-n_{3}} a_{i}^{K_{1}-n_{3}} e^{\alpha_{i}^{K_{1}-n_{3}} t}\right. \\
& \left.\times \sum_{j=1}^{K_{2}+1-n_{3}} b_{j}^{K_{2}-n_{3}} e^{\beta_{j}^{K_{2}-n_{3}} t}\right][\text { by }(6)] \\
= & 1-\mathbf{V}^{T}(t) \cdot \mathbf{P}_{N_{3}}(t)
\end{aligned}
$$

where

$$
\begin{aligned}
& \mathbf{V}(t)=\left[\begin{array}{c}
\sum_{i=1}^{K_{1}+1} \sum_{j=1}^{K_{2}+1} a_{i}^{K_{1}} b_{j}^{K_{2}} e^{\left[\alpha_{i}^{K_{1}}+\beta_{j}^{K_{2}}\right] t} \\
\sum_{i=1}^{K_{1}} \sum_{j=1}^{K_{2}} a_{i}^{K_{1}-1} b_{j}^{K_{2}-1} e^{\left[\alpha_{i}^{K_{1}-1}+\beta_{j}^{K_{2}-1}\right] t} \\
\vdots
\end{array}\right] \\
& \mathbf{P}_{N_{3}}(t)=\left[\begin{array}{c}
P_{\left(N_{3}=0\right)}(t) \\
\vdots \\
P_{\left(N_{3}=K_{m}\right)}(t)
\end{array}\right] \text {. }
\end{aligned}
$$

$\alpha_{i}^{K_{1}-n_{3}}$ and $\beta_{j}^{K_{2}-n_{3}}$ are the eigenvalues of link-1 and link-2 of capacity $K_{1}-n_{3}$ and $K_{2}-n_{3}$, respectively. $a_{i}^{K_{1}-n_{3}}$ and $b_{j}^{K_{2}-n_{3}}$ are the corresponding coefficients. 


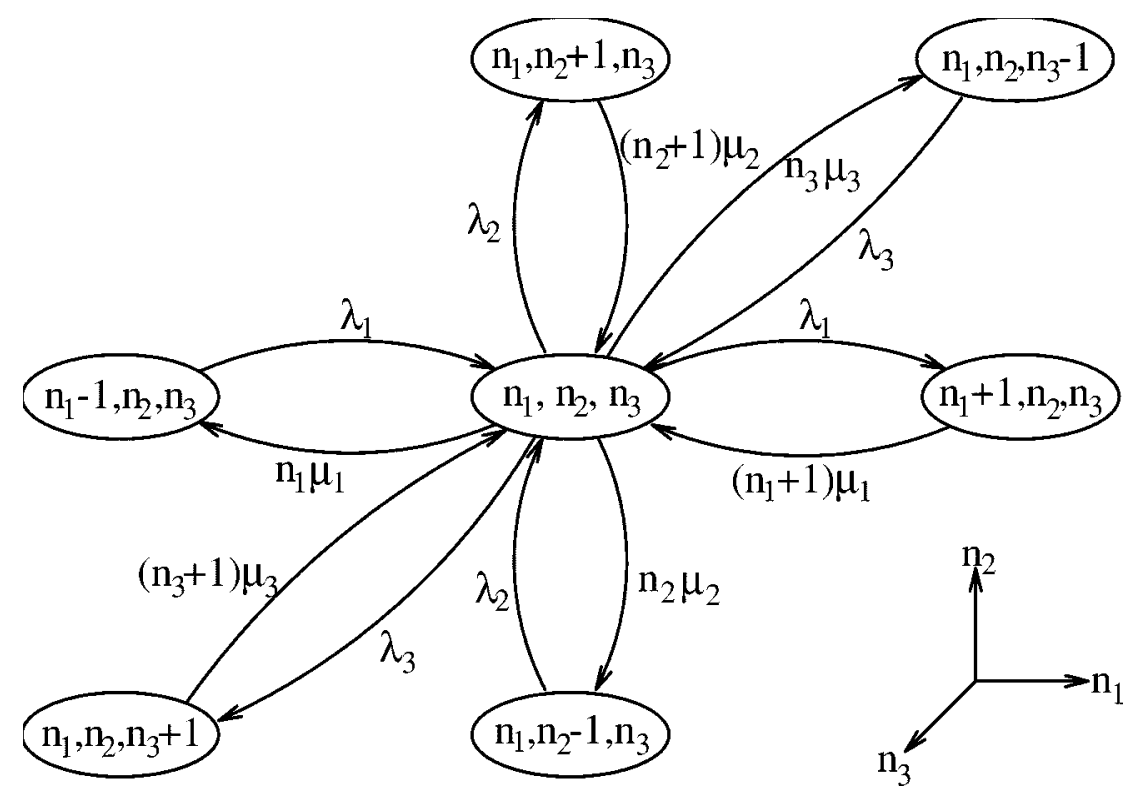

Fig. 16. State transition diagram of the 3-D Markov chain for a two-link network.

Now we calculate $\mathbf{P}_{N_{3}}(t)$ at time $t$

$$
\begin{aligned}
P_{\left(N_{3}=n_{3}\right)}(t)= & \sum_{n_{1}=0}^{K_{1}+1-n_{3}} \sum_{n_{2}=0}^{K_{2}+1-n_{3}} P_{n_{1}, n_{2}, n_{3}}(t) \\
\text { So } P_{\left(N_{3}=n_{3}\right)}^{\prime}(t)= & \sum_{n_{1}=0}^{K_{1}-n_{3}} \sum_{n_{2}=0}^{K_{2}-n_{3}} P_{n_{1}, n_{2}, n_{3}}^{\prime}(t) \\
& +\sum_{n_{1}=0}^{K_{1}-n_{3}} P_{n_{1}, K_{2}+1-n_{3}, n_{3}}^{\prime}(t) \\
& +\sum_{n_{2}=0}^{K_{2}-n_{3}} P_{K_{1}+1-n_{3}, n_{2}, n_{3}}^{\prime}(t) \\
& +P_{K_{1}+1-n_{3}, K_{2}+1-n_{3}, n_{3}}^{\prime}(t) .
\end{aligned}
$$

Substituting the expressions from (24) and (25) for transient and absorbing states, respectively, and simplifying the resulting expression we get the following simple form:

$$
\begin{aligned}
P_{\left(N_{3}=n_{3}\right)}^{\prime}(t)= & -\left(\lambda_{3}+n_{3} \mu_{3}\right) \sum_{n_{1}=0}^{K_{1}-n_{3}} \sum_{n_{2}=0}^{K_{2}-n_{3}} P_{n_{1}, n_{2}, n_{3}}(t) \\
& +f\left(n_{3}\right) \lambda_{3} \sum_{n_{1}=0}^{K_{1}+1-n_{3}} \sum_{n_{2}=0}^{K_{2}+1-n_{3}} P_{n_{1}, n_{2}, n_{3}-1}(t) \\
& +I_{\left\{n_{3}<K_{m}\right\}}\left(n_{3}+1\right) \mu_{3} \\
& \times \sum_{n_{1}=0}^{K_{1}-1-n_{3}} \sum_{n_{2}=0}^{K_{2}-1-n_{3}} P_{n_{1}, n_{2}, n_{3}+1}(t) \\
= & -\left(\lambda_{3}+n_{3} \mu_{3}\right) P_{\left(\text {noabs } \mid N_{3}=n_{3}\right)}(t) P_{\left(N_{3}=n_{3}\right)}(t) \\
& +f\left(n_{3}\right) \lambda_{3} P_{\left(\text {noabs } \mid N_{3}=n_{3}-1\right)}(t) P_{\left(N_{3}=n_{3}-1\right)}(t) \\
& +I_{\left\{n_{3}<K\right\}}\left(n_{3}+1\right) \mu_{3} \\
& \times P_{\left(\text {noabs } \mid N_{3}=n_{3}+1\right)}(t) P_{\left(N_{3}=n_{3}+1\right)}(t)
\end{aligned}
$$

where

$P_{\left(\text {noabs } \mid N_{3}=n_{3}\right)}(t)=P_{\text {noabs }}^{(1)}\left(K_{1}-n_{3}, t\right) \cdot P_{\text {noabs }}^{(2)}\left(K_{2}-n_{3}, t\right)$.

So $\mathbf{P}_{N_{3}}(t)$ can be calculated from the following system of differential equations with time-dependent coefficients:

$$
\mathbf{P}_{N_{3}}^{\prime}(t)=\mathbf{M D}(t) \mathbf{P}_{N_{3}}(t)
$$

with $\mathbf{P}_{N_{3}}(0)=\left[\begin{array}{lllll}1 & 0 & 0 & \cdots & 0\end{array}\right]^{T}$.

$\mathbf{M}$ is a constant tridiagonal matrix and $\mathbf{D}(t)$ is a variable diagonal matrix.

These types of differential equations can be solved easily by numerical methods. Some common methods are based on Taylor series expansion.

\section{REFERENCES}

[1] R. Ramaswami, "Multi-wavelength lightwave networks for computer communication," IEEE Commun. Mag., vol. 31, pp. 78-88, Feb. 1993.

[2] R. Ramaswami and K. N. Sivarajan, Optical Networks: A Practical Perspective. San Fransisco, CA: Morgan Kaufmann, 1998.

[3] S. Nash and A. Sofer, Linear and Nonlinear Programming. New York: McGraw-Hill, 1996.

[4] D. G. Luenberger, Linear and Nonlinear Programming: Addison-Wesley, 1984.

[5] P. E. Gill, W. Murry, and M. Wright, Practical Optimization. New York: Academic, 1981.

[6] A. Girard, Routing and Dimensioning in Circuit-Switched Networks: Addison-Wesley, 1990.

[7] A. Girard and R. Page, "Dimensioning of telephone networks with nonhierarchical routing and trunk reservation," in Network Planning Symp., vol. 3, June 1986, pp. 85-93.

[8] R. J. Harris, "Comparision of network dimensioning models," Australian Telecommunication Res., vol. 18, pp. 59-69, 1984.

[9] M. P. Pioro, "A uniform approach to the analysis and optimization of circuit switched communication networks," in Int. Teletraffic Congr., vol. 10, 1983, pp. 4.3A:1-4.3A:7.

[10] S. Katz, "Trunk engineering of nonhierarchical networks," in Int. Teletraffic Congr., vol. 6, 1971, pp. 142.1-142.8. 
[11] F. P. Kelly, "Blocking probabilities in large circuit-switched networks," Advances in Applied Probability, vol. 18, pp. 473-505, 1986.

[12] A. Birman, "Computing approximate blocking probabilities for a class of all-optical networks," IEEE J. Select. Areas Commun., vol. 14, pp. 852-857, June 1996.

[13] A. Sridharan and K. N. Sivarajan, "Blocking in all-optical networks," Proc. IEEE INFOCOM 2000, Mar. 2000.

[14] T. Tripathi and K. N. Sivarajan, "Computing approximate blocking probabilities for wavelength-routed all-optical networks with limited-wavelength conversion," Proc. IEEE INFOCOM '99.

[15] W. Feller, An Introduction to Probability Theory and Its Applications, 3rd ed. New York: Wiley, 1968, vol. 1, pp. 454-457.

[16] A. Papoulis, Probability, Random Variables and Stochastic Processes, 3rd ed. New York: McGraw-Hill, 1991.

[17] R. A. Frazer, W. J. Duncan, and A. R. Collar, Elementary Matrices. Oxford, U.K.: Cambridge Univ. Press, 1938.

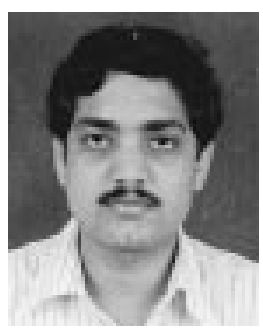

Tapan Kumar Nayak (S'01) received the B.E. degree in electronics and telecommunication engineering from the Jadavpur University, Calcutta, in 1996, and the M.E. degree in electrical communication engineering from the Indian Institute of Science, Bangalore, in 1998. Since 1999, he has been pursuing the $\mathrm{Ph} . \mathrm{D}$. degree at the Indian Institute of Science.

His research interests include network architectures, queuing theory, and video communications.

Mr. Nayak was the recipient of a scholarship from LG Electronics, Korea, in 1997, and the IBM Research Fellowship in 1999.

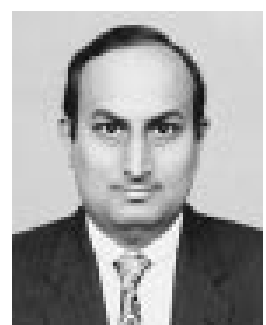

Kumar N. Sivarajan (M'88) received the B.Tech. degree in electrical engineering (electronics) from the Indian Institute of Technology, Madras, in 1987, and the M.S. and Ph.D. degrees in electrical engineering from the California Institute of Technology, Pasadena, in 1988 and 1990, respectively.

From 1990 to 1994, he was with IBM T. J. Watson Research Center, Yorktown Heights, NY. From 1994 to 2000, he was with with the Electrical Communication Engineering Department, Indian Institute of Science, Bangalore. Since May 2000, he has been Chief Technology Officer of Tejas Networks, Bangalore.

Dr. Sivarajan has served as an Editor of the IEEE/ACM TRANSACTIONS ON NETWORKING. He is an Associate of the Indian Academy of Sciences and the recipient of the Young Engineer Award from the Indian National Academy of Engineering and the Swarnajayanti Fellowship from the Department of Science and Technology, Government of India. He was the recipient of the IEEE Charles LeGeyt Fortescue Fellowship, for the academic year 1987 to 1988, the IEEE Communications Society 1996 William R. Bennett Prize Paper Award, and the 1997 IEEE W. R. G. Baker Prize Paper Award. 